\title{
A unified approach to a priori estimates for supersolutions of BSDEs in general filtrations
}

\author{
Bruno Bouchard $^{\mathrm{a}}$, Dylan Possamaï ${ }^{\mathrm{a}}$, Xiaolu Tan ${ }^{\mathrm{a}, 1}$ and Chao Zhou ${ }^{\mathrm{b}, 2}$ \\ ${ }^{a}$ Université Paris-Dauphine, PSL Research University, CNRS, UMR [7534], Ceremade, 75016 Paris, France. ANR Liquirisk. \\ E-mail: bouchard@ceremade.dauphine.fr; possamai@ceremade.dauphine.fr; tan@ceremade.dauphine.fr \\ ${ }^{\mathrm{b}}$ Department of Mathematics, National University of Singapore, Singapore. E-mail: matzc@nus.edu.sg
}

Received 24 July 2015; revised 15 September 2016; accepted 26 September 2016

\begin{abstract}
We provide a unified approach to a priori estimates for supersolutions of BSDEs in general filtrations, which may not be quasi left-continuous. Unlike the previous related approaches in simpler settings, our results do not only rely on a simple application of Itô's formula and classical estimates, but use crucially appropriate generalizations of deep estimates for supermartingales obtained by Meyer. As an example of application, we prove that reflected BSDEs are well-posed in a general framework which has not been covered so far in the existing literature.
\end{abstract}

Résumé. Nous proposons dans cet article une approche unifiée permettant l'obtention d'estimées a priori pour des sur-solutions d'EDSR adaptées à des filtrations générales, en particulier non nécessairement quasi-continues à gauche. Contrairement aux approches antérieures de ce problème dans des cadres plus simples, nos résultats ne sont pas la conséquence directe de la formule d'Itô et d'estimées classiques, mais dépendent de manière cruciale de versions appropriées à notre contexte d'estimées obtenues par Meyer pour des sur-martingales. Nous proposons entre autres une application de nos résultats à l'étude de l'existence et de l'unicité de solutions d'EDSR réfléchies dans un cadre général non-couvert par les résultats précédents dans la littérature.

MSC: $60 \mathrm{H} 99$

Keywords: Backward SDE; Supersolution; Doob-Meyer decomposition; Reflected backward SDE

\section{Introduction}

Supersolutions of backward stochastic differential equations (BSDEs from now on) were introduced by El Karoui et al. in their seminal paper [9], in order to study superhedging strategies in mathematical finance. In the simple context of a filtered probability space $\left(\Omega, \mathcal{F}, \mathbb{F}:=\left(\mathcal{F}_{t}\right)_{0 \leq t \leq T}, \mathbb{P}\right)$ where $\mathbb{F}$ is the (augmented) natural filtration of a $d$-dimensional Brownian motion $W$, a supersolution of a BSDE with terminal condition $\xi$ and generator $g$ consists in a triple of $\mathbb{F}$-adapted processes $(Y, Z, K)$, living in appropriate spaces, with $K$ predictable non-decreasing, such that

$$
Y_{t}=\xi-\int_{t}^{T} g_{s}\left(Y_{s}, Z_{s}\right) d s-\int_{t}^{T} Z_{s} \cdot d W_{s}+\int_{t}^{T} d K_{s}, \quad t \in[0, T], \mathbb{P} \text {-a.s. }
$$

These objects appeared later to be at the very heart of the study of reflected BSDEs, as introduced in El Karoui et al. [8], and more generally of BSDEs satisfying some constraint, see Cvitanić, Karatzas and Soner [5] for constraints

\footnotetext{
${ }^{1}$ Supported by ERC 321111 Rofirm, the ANR Isotace, and the Chairs Financial Risks (Risk Foundation, sponsored by Société Générale) and Finance and Sustainable Development (IEF sponsored by EDF and CA).

${ }^{2}$ Supported by NUS Grants R-146-000-179-133 and R-146-000-219-112.
} 
on the $Z$-component and Peng et al. [18,23-26] for general restrictions. More recently, supersolutions of BSDEs have been proved to provide the semimartingale decomposition of the so-called second order BSDEs, introduced by Soner, Touzi and Zhang [29] and generalized by Possamaï, Tan and Zhou [27], and of the weak BSDEs studied by Bouchard, Élie and Réveillac in [1].

When the generator $g$ is equal to 0 , the process $Y$ defined above is nothing else but a supermartingale, and (1.1) is simply its Doob-Meyer decomposition. This was generalized by Peng [23] using the notion of non-linear supermatingales, see also $[2,10]$ and the references therein.

As seen through the above examples, supersolutions of BSDEs appear quite frequently in the literature, as natural semimartingale decompositions for various stochastic processes, and are often used to study their fine properties. Having at hand a priori estimates on the moments and on the stability of supersolutions is crucial in these contexts. Unfortunately, in almost all the previously cited works, with the exception of [27], such estimates have been written in, roughly speaking, the context of a Brownian filtration. This is rather limiting from the point of view of both the theory and the applications, and it has created a tendency in the recent literature to reproduce very similar proofs every time that the context was generalized.

In this paper, we propose a general approach which allows one to consider a quite sufficiently general setting. In particular, we do not assume that the underlying filtration is generated by a Brownian motion. In this case, one needs to introduce another component in the definition of a supersolution of a BSDE, namely a martingale $M$ that is orthogonal to $W$ :

$$
Y_{t}=\xi-\int_{t}^{T} g_{s}\left(Y_{s}, Z_{s}\right) d s-\int_{t}^{T} Z_{s} \cdot d W_{s}-\int_{t}^{T} d M_{s}+\int_{t}^{T} d K_{s}, \quad t \in[0, T], \mathbb{P} \text {-a.s. }
$$

When $K \equiv 0$, such objects were first introduced by El Karoui and Huang [7], and studied more recently by Kruse and Popier [14] to handle more general filtrations, in the context of $\mathbb{L}^{p}$-solutions, as in the seminal papers $[3,9]$. Supersolutions in general filtrations play a crucial role for the class of reflected BSDEs studied by Klimsiak [13], which is, as far as we know, the most general reference to date. However, all [7,13], ${ }^{3}$ [14] still impose that the filtration is quasi left-continuous, a property which, for instance, is not satisfied for the second order BSDEs studied in [27]. We remind the reader that the filtration $\mathbb{F}$, assumed to satisfy the usual hypotheses, is said to be quasi-left continuous if, for any $\mathbb{F}$-predictable stopping time $\tau$, one has

$$
\mathcal{F}_{\tau}=\mathcal{F}_{\tau-}, \quad \text { where } \mathcal{F}_{\tau-}:=\sigma\left(A \cap\{t<\tau\}, A \in \mathcal{F}_{t}\right) \vee \mathcal{N}
$$

where $\mathcal{N}$ is the the set of all null sets in $(\Omega, \mathcal{F}, \mathbb{P})$. Intuitively, this means that martingales with respect to $\mathbb{F}$ cannot have predictable times of jumps, and in particular deterministic times of jumps.

To understand the simplifications induced by the quasi left-continuity assumption, let us give a brief sketch of the strategy of proof usually used to obtain estimates, say in $\mathbb{L}^{2}$ for simplicity:

(i) Apply Itô's formula to $e^{\alpha \cdot} Y^{2}$ to obtain

$$
\begin{aligned}
& e^{\alpha t} Y_{t}^{2}+\alpha \int_{t}^{T} e^{\alpha s} Y_{s}^{2} d s+\int_{t}^{T} e^{\alpha s}\left\|Z_{s}\right\|^{2} d s+\int_{t}^{T} e^{\alpha s} d[M]_{s} \\
& \quad=e^{\alpha T} \xi^{2}-2 \int_{t}^{T} e^{\alpha s} Y_{s} g_{s}\left(Y_{s}, Z_{s}\right) d s-2 \int_{t}^{T} e^{\alpha s} Y_{s} Z_{s} \cdot d W_{s}-2 \int_{t}^{T} e^{\alpha s} Y_{s-} d M_{s}+2 \int_{t}^{T} e^{\alpha s} Y_{s-} d K_{s} .
\end{aligned}
$$

(ii) Take expectations on both sides, use classical inequalities (namely Young and Burkholder-Davis-Gundy) and some continuity assumptions on $g$ (usually Lipschitz continuity) to control $\mathbb{L}^{2}$-type norms of $(Y, Z, M)$ by the norm of $K$ times a small constant, when $\alpha>0$ is large enough.

(iii) Use the definition of a supersolution to control the norm of $K$ by the norms of $(Y, Z, M)$, and conclude.

\footnotetext{
${ }^{3}$ Notice that in [13], when the generator does not depend on $Z$ and $p=1$, there is no need for the quasi left-continuity assumption. But the general case requires it.
} 
What is actually hidden in this reasoning is that, because the martingale $M$ cannot jump at predictable times when the filtration is quasi left-continuous, the bracket $[M, K]$ is identically equal to 0 . This is no longer true for general filtrations, in which case we have to deal with the term $[M, K]$. It turns out to be difficult to control, which makes this traditional approach not amenable to filtrations that are not quasi left-continuous.

In the case where we look for estimates in $\mathbb{L}^{2}$, this is not a problem because this bracket term is indeed a (local) martingale, see [28, proof of Lemma 6], ${ }^{4}$ and taking expectations in both sides (up to localization) is enough. This does not work anymore for $p \neq 2$ because then the formulation corresponding to (1.3) involves a non-linear transformation of this martingale.

Of course, such a problem only appears when one considers supersolutions, which is not the case in [7] or [13]. The main problem in [7] is that since they consider BSDEs driven by a general càdlàg martingale $N$, the generator $g$ is integrated with respect to a Stieljes measure related to $d\langle N\rangle$, and, if the filtration is not quasi-left continuous, then $\langle N\rangle$ may have jumps in general, which prevents the technics in [7] to be applied. In [14] however, the problem comes from the fact that they consider so-called BSDEs with jumps, which adds another martingale in the definition of the BSDE. When the filtration is not quasi-left continuous, this martingale can jump at predictable times, which makes the analysis more difficult.

The main aim of our paper is to give a general proof of a priori estimates and stability for supersolutions of BSDEs in a possibly non-quasi-left-continuous filtration. The proof relies on the following property:

"It is sufficient to control the norm of $Y$ to control the norm of $(Y, Z, M, K)$."

This is the philosophy of the estimates of Meyer [21, Theorem 1] that apply to general super-martingales (see also the generalization in [16, Theorem 3.1]). In Section 2, we show how it can be generalized to the non-linear context of BSDEs. Namely, Theorem 2.1 below provides the extension of [21, Theorem 1] to a supersolution, while Theorem 2.2 is a version that applies to the difference of two supersolutions. Both are valid for supersolutions that are only làdlàg. In Section 3, we use these results to provide a well-posedness result for reflected BSDEs with a càdlàg obstacle. When there is no quasi left-continuity assumption on the filtration, this result is not available in the existing literature.

\section{Notations}

For any $l \in \mathbb{N} \backslash\{0\}$, we denote the usual inner product of two vectors $(x, y) \in \mathbb{R}^{l} \times \mathbb{R}^{l}$ by $x \cdot y$. The Euclidean norm on $\mathbb{R}^{l}$ is denoted by $\|\cdot\|$, and simplified to $|\cdot|$ when $l=1$. Let $T>0$ be fixed and let $(\Omega, \mathcal{F}, \mathbb{P})$ be a complete probability space, equipped with a filtration $\mathbb{F}=\left(\mathcal{F}_{t}\right)_{0 \leq t \leq T}$ satisfying the usual conditions, and carrying a standard $d$-dimensional $\mathbb{F}$-Brownian motion $W$. Importantly, we do not assume that the filtration is quasi left-continuous. Given $p>1$ and $\alpha>0$, we introduce the classical spaces:

- $\mathbb{L}^{p}$ is the space of $\mathbb{R}$-valued and $\mathcal{F}_{T}$-measurable random variables $\xi$ such that

$$
\|\xi\|_{\mathbb{L}^{p}}^{p}:=\mathbb{E}\left[|\xi|^{p}\right]<+\infty .
$$

- $\mathbb{S}^{p}$ (resp. $\mathbb{S}_{r}^{p}$ ) denotes the space of $\mathbb{R}$-valued, $\mathbb{F}$-adapted processes $Y$, with $\mathbb{P}$-a.s. làdlàg (resp. càdlàg) paths, such that

$$
\|Y\|_{\mathbb{S} p}^{p}:=\mathbb{E}\left[\sup _{0 \leq s \leq T}\left|Y_{t}\right|^{p}\right]<+\infty .
$$

- $\mathbb{M}^{p, \alpha}$ is the space of $\mathbb{R}$-valued, $\mathbb{F}$-adapted martingales $M$, with $\mathbb{P}$-a.s. càdlàg paths, such that $M$ is orthogonal to $W$ and

$$
\|M\|_{\mathbb{M} p, \alpha}^{p}:=\mathbb{E}\left[\left(\int_{0}^{T} e^{\alpha s} d[M]_{s}\right)^{\frac{p}{2}}\right]<+\infty .
$$

\footnotetext{
${ }^{4}$ We thank M. Schweizer for pointing this argument out to us.
} 
- $\mathbb{H}^{p, \alpha}$ (resp. $\mathbb{H}_{1}^{p, \alpha}$ ) is the space of $\mathbb{R}^{d}$-valued (resp. $\mathbb{R}$-valued) and $\mathbb{F}$-predictable processes $Z$ such that

$$
\|Z\|_{\mathbb{H} p, \alpha}^{p}:=\mathbb{E}\left[\left(\int_{0}^{T} e^{\alpha s}\left\|Z_{s}\right\|^{2} d s\right)^{\frac{p}{2}}\right]<+\infty .
$$

- $\mathbb{I}^{p, \alpha}$ (resp. $\mathbb{I}_{+}^{p, \alpha}, \mathbb{I}_{r}^{p, \alpha}, \mathbb{I}_{+, r}^{p, \alpha}$ ) denotes the space of $\mathbb{R}$-valued, $\mathbb{F}$-predictable processes with bounded variations $K$, with $\mathbb{P}$-a.s. làdlàg (resp. non-decreasing làdlàg, càdlàg, non-decreasing càdlàg) paths, such that

$$
\|K\|_{\mathbb{I} p, \alpha}^{p}:=\mathbb{E}\left[\left(\int_{0}^{T} e^{\frac{\alpha}{2} s} d \operatorname{TV}(K)_{s}\right)^{p}\right]<+\infty
$$

and $K_{0}=0$. In the above $\operatorname{TV}(K)$ denotes the total variation of $K$.

- We also define $\mathbb{S}_{\text {loc }}^{p}$ as the collection of processes $Y$ such that, for an increasing sequence of stopping times $\left(\tau_{n}\right)_{n \geq 1}$ satisfying $\mathbb{P}\left(\lim _{n \rightarrow \infty} \tau_{n}=\infty\right)=1$, the localized process $Y_{\tau_{n} \wedge}$. belongs to $\mathbb{S}^{p}$ for each $n \geq 1$. The spaces $\mathbb{M}_{\text {loc }}^{p, \alpha}$, $\mathbb{H}_{\text {loc }}^{p, \alpha}, \mathbb{H}_{1, \text { loc }}^{p, \alpha}, \mathbb{I}_{\text {loc }}^{p, \alpha}$, and $\mathbb{I}_{+, \text {loc }}^{p, \alpha}$ are defined similarly.

- Finally, for $\alpha=0$, we simplify the notation $\mathbb{M}^{p}:=\mathbb{M}^{p, 0}, \mathbb{H}^{p}:=\mathbb{H}^{p, 0}, \mathbb{H}_{1}^{p}:=\mathbb{H}_{1}^{p, 0}, \mathbb{I}^{p}:=\mathbb{I}^{p, 0}, \mathbb{I}_{+}^{p}:=\mathbb{I}_{+}^{p, 0}, \mathbb{I}_{r}^{p}:=$ $\mathbb{I}_{r}^{p, 0}$ and $\mathbb{I}_{+, r}^{p}:=\mathbb{I}_{+, r}^{p, 0}$.

Note that the above spaces do not depend on the precise value of $\alpha$ as we work on the compact time interval $[0, T]$, two values of $\alpha$ actually provide equivalent norms. Still, we keep the parameter $\alpha$ which, as usual, will be very helpful for many of our arguments.

Given a làdlàg optional process $X$, such that its right-limit process $X^{+}$is a semimartingale, and a locally bounded predictable process $\phi$, we define the stochastic integral as in [15]:

$$
(\phi \star X)_{t}:=\int_{0}^{t} \phi_{s} d X_{s}:=\int_{0}^{t} \phi_{s} d X_{s}^{+}-\phi_{t}\left(X_{t+}-X_{t}\right), \quad t \geq 0 .
$$

Moreover, we define $\int_{t}^{T} \phi_{s} d X_{s}:=\int_{0}^{T} \phi_{s} d X_{s}-\int_{0}^{t} \phi_{s} d X_{s}$.

\section{A priori estimates}

Let us consider a BSDE with terminal condition $\xi$ and generator $g:[0, T] \times \Omega \times \mathbb{R} \times \mathbb{R}^{d} \longrightarrow \mathbb{R}$. For ease of notations, we denote $g_{t}^{0}(\omega):=g_{t}(\omega, 0,0)$. Although, we will have to differentiate between possible values of $p>1$, this parameter is fixed from now on. The following standing assumption is assumed throughout this section.

\section{Assumption 2.1.}

(i) $\xi \in \mathbb{L}^{p}, g^{0} \in \mathbb{H}_{1}^{p}$ and the process $(t, \omega) \longmapsto g_{t}(\omega, y, z)$ is $\mathbb{F}$-progressively measurable for all $(y, z) \in \mathbb{R} \times \mathbb{R}^{d}$.

(ii) There exist $\left(L_{y}, L_{z}\right) \in \mathbb{R}_{+}^{2}$, independent of any variables, s.t. for all $\left(t, \omega, y_{1}, z_{1}, y_{2}, z_{2}\right) \in[0, T] \times \Omega \times\left(\mathbb{R} \times \mathbb{R}^{d}\right)^{2}$

$$
\left|g_{t}\left(\omega, y_{1}, z_{1}\right)-g_{t}\left(\omega, y_{2}, z_{2}\right)\right| \leq L_{y}\left|y_{1}-y_{2}\right|+L_{z}\left\|z_{1}-z_{2}\right\| .
$$

We recall here the definition of a supersolution.

Definition 2.1. We say that $(Y, Z, M, K)$ is a solution (resp. local solution) of

$$
Y_{t}=\xi-\int_{t}^{T} g_{s}\left(Y_{s}, Z_{s}\right) d s-\int_{t}^{T} Z_{s} \cdot d W_{s}-\int_{t}^{T} d M_{s}+K_{T}-K_{t},
$$

if the above holds for any $t \in[0, T]$, $\mathbb{P}$-a.s., and $(Y, Z, M, K) \in \mathbb{S}^{p} \times \mathbb{H}^{p} \times \mathbb{M}^{p} \times \mathbb{I}^{p}$ (resp. $(Y, Z, M, K) \in \mathbb{S}_{\text {loc }}^{p} \times$ $\mathbb{H}_{\mathrm{loc}}^{p} \times \mathbb{M}_{\mathrm{loc}}^{p} \times \mathbb{I}_{\mathrm{loc}}^{p}$ ). If moreover $K \in \mathbb{I}_{+}^{p}$ (resp. $\mathbb{I}_{+, \text {loc }}^{p}$ ), we say that $(Y, Z, M, K)$ is a supersolution (resp. a local supersolution) of (2.2). 


\subsection{Estimates for the solution}

Our main result says that one can control $(Z, M, K)$ by controlling the component $Y$ of a solution $(Y, Z, M, K)$. We emphasize that the general setting we consider here creates additional difficulties that have not been tackled so far in the literature, and which mainly stems from the fact that it is possible for the processes $K$ and $M$ to jump at the same time, when the filtration is not quasi left-continuous. Therefore, the traditional approach which consists in applying Itô's formula to $|Y|^{p}$ to derive the desired estimates fails, as this makes the cross-variation between $M$ and $K$ appear, a term that has no particular sign and cannot be controlled easily. Our message here is that, in order to obtain such estimates in a general setting, one should rely on a deeper result from the general theory of processes, namely the estimates obtained in Meyer [21] for general supermartingales, a version of which we recall in the Appendix below, see Lemma A.1.

The following is an extension to the non-linear context.

Theorem 2.1. Let $(Y, Z, M, K) \in \mathbb{S}^{p} \times \mathbb{H}^{p} \times \mathbb{M}^{p} \times \mathbb{I}_{+}^{p}$ be a solution of (2.2). Then, for any $\alpha \geq 0$, there is a constant $C_{2.1}^{\alpha}$ such that

$$
\|Z\|_{\mathbb{H} p, \alpha}^{p}+\|M\|_{\mathbb{M} p, \alpha}^{p}+\|K\|_{\mathbb{I} p, \alpha}^{p} \leq C_{2.1}^{\alpha}\left(\|\xi\|_{\mathbb{L}^{p}}^{p}+\|Y\|_{\mathbb{S} p}^{p}+\left\|g^{0}\right\|_{\mathbb{H}_{1}^{p, \alpha}}^{p}\right)
$$

Before proving this result, we shall establish more general intermediate estimates, that will also be used to control the difference of solutions in Theorem 2.2 below. They use the notation

$$
N:=Z \star W+M-K .
$$

We start with an easy remark.

Remark 2.1. (i) First note that for any $\ell>0$ and $\left(a_{i}\right)_{1 \leq i \leq n} \subset(0,+\infty)$,

$$
\left(1 \wedge n^{\ell-1}\right) \sum_{i=1}^{n} a_{i}^{\ell} \leq\left(\sum_{i=1}^{n} a_{i}\right)^{\ell} \leq\left(1 \vee n^{\ell-1}\right) \sum_{i=1}^{n} a_{i}^{\ell} .
$$

Let us now consider a solution $(Y, Z, M, K) \in \mathbb{S}^{p} \times \mathbb{H}^{p} \times \mathbb{M}^{p} \times \mathbb{I}^{p}$ of (2.2). Since $W$ and $M$ are orthogonal, (2.3) implies that

$$
\left(1 \wedge 2^{\frac{p}{2}-1}\right)\left(\|Z\|_{\mathbb{H} p, \alpha}^{p}+\|M-K\|_{\mathbb{M}^{p, \alpha}}^{p}\right) \leq\|N\|_{\mathbb{M}^{p, \alpha}}^{p} \leq\left(1 \vee 2^{\frac{p}{2}-1}\right)\left(\|Z\|_{\mathbb{H} p, \alpha}^{p}+\|M-K\|_{\mathbb{M}^{p, \alpha}}^{p}\right) .
$$

Moreover, if $K \in \mathbb{I}_{+}^{p, \alpha}$ then the Kunita-Watanabe inequality leads to

$$
d[M+Z \star W] \leq 2(d[N]+d[K]) \leq 2\left(d[N]+2 K_{-} d K+d[K]\right) \leq 2\left(d[N]+d K^{2}\right),
$$

so that by $(2.3)$

$$
\begin{aligned}
\|M+Z \star W\|_{\mathbb{M} p, \alpha}^{p} & \leq 2^{\frac{p}{2}} \mathbb{E}\left[\left(\int_{0}^{T} e^{\alpha s}\left(d[N]_{s}+d K_{s}^{2}\right)\right)^{\frac{p}{2}}\right] \\
& \leq 2^{\frac{p}{2}}\left(1 \vee 2^{\frac{p}{2}-1}\right)\left(\|N\|_{\mathbb{M} p, \alpha}^{p}+e^{\frac{\alpha p T}{2}}\|K\|_{\mathbb{I} p, \alpha}^{p}\right) .
\end{aligned}
$$

Hence, since $W$ and $M$ are orthogonal, we finally have

$$
\left(1 \wedge 2^{\frac{p}{2}-1}\right)\left(\|M\|_{\mathbb{M} p, \alpha}^{p}+\|Z\|_{\mathbb{H} p, \alpha}^{p}\right) \leq\|M+Z \star W\|_{\mathbb{M}^{p, \alpha}}^{p} \leq\left(2^{\frac{p}{2}} \vee 2^{p-1}\right)\left(\|N\|_{\mathbb{M} p, \alpha}^{p}+e^{\frac{\alpha p T}{2}}\|K\|_{\mathbb{I} p, \alpha}^{p}\right),
$$

in which the left-hand side inequality remains true even if $K$ is not non-decreasing. 
(ii) In the following, we shall also use the standard Young's inequality

$$
a b \leq \beta a^{p}+\frac{b^{q}}{q(\beta p)^{q / p}}, \quad \text { for } a, b \in \mathbb{R}_{+}, \beta>0, p, q>1 \text { and } \frac{1}{p}+\frac{1}{q}=1 .
$$

(iii) We also emphasize that for $(Y, Z, M) \in \mathbb{S}^{p} \times \mathbb{H}^{p} \times \mathbb{M}^{p}$, the process

$$
\int_{0}^{\cdot} e^{p \frac{\alpha}{2} s} \phi_{p}\left(Y_{s-}\right) d(M+Z \star W),
$$

is a uniformly integrable martingale, where

$$
\phi_{p}(y)=|y|^{p-1} \operatorname{sgn}(y) \mathbf{1}_{y \neq 0}, \quad \text { for } y \in \mathbb{R} .
$$

Indeed, Burkholder-Davis-Gundy and Hölder's inequalities imply

$$
\begin{aligned}
& \mathbb{E}\left[\sup _{0 \leq t \leq T}\left|\int_{0}^{t} e^{p \frac{\alpha}{2} s} \phi_{p}\left(Y_{s-}\right) d(M+Z \star W)_{s}\right|\right] \\
& \leq C \mathbb{E}\left[\sqrt{\int_{0}^{T} e^{p \alpha s}\left|Y_{S-}\right|^{2 p-2} d[M]_{s}+\int_{0}^{T} e^{p \alpha s}\left|Y_{S}\right|^{2 p-2}\left\|Z_{S}\right\|^{2} d s}\right] \\
& \leq C e^{(p-1) \frac{\alpha}{2} T}\|Y\|_{\mathbb{S} p}^{p-1}\left(\|M\|_{\mathbb{M} p, \alpha}+\|Z\|_{\mathbb{H}}^{p, \alpha}\right),
\end{aligned}
$$

for some $C>0$.

From now on, we use the generic notation $C$, combined with super- and subscripts, to denote constants in our estimates that only depend on $L_{y}, L_{z}, p$ and $\alpha$. If they depend on other parameters, this will be made clear. Although we do not provide their expressions explicitly, our proofs are written in such a way that the interested reader can easily keep track of them line after line.

In the following, the inequality (2.8) is the crucial one, this is the consequence of Meyer [21].

Lemma 2.1. Let $(Y, Z, M, K) \in \mathbb{S}^{p} \times \mathbb{H}^{p} \times \mathbb{M}^{p} \times \mathbb{I}^{p}$ be a solution of (2.2).

(i) If $K \in \mathbb{I}_{+}^{p}$, then for all $\alpha \geq 0$ there exists a constant $C_{(2.8)}^{\alpha}$ such that

$$
\|K\|_{\mathbb{I} p, \alpha}^{p} \leq C_{(2.8)}^{\alpha}\left(\left\|e^{\frac{\alpha}{2} \cdot Y}\right\|_{\mathbb{S} p}^{p}+\|Z\|_{\mathbb{H} p, \alpha}^{p}+\left\|g^{0}\right\|_{\mathbb{H}_{1}^{p, \alpha}}^{p}\right) .
$$

(ii) If $p \geq 2$, then for all $\varepsilon>0$ there exists $\alpha>0$ and a constant $C_{(2.9)}^{\varepsilon, \alpha}$ such that

$$
\|Y\|_{\mathbb{H}_{1}^{p, \alpha}}^{p}+\|N\|_{\mathbb{M}^{p, \alpha}}^{p} \leq \varepsilon\left\|g^{0}\right\|_{\mathbb{H}_{1}^{p, \alpha}}^{p}+C_{(2.9)}^{\varepsilon, \alpha}\left(\|\xi\|_{\mathbb{L}^{p}}^{p}+\left\|\left(e^{\alpha \cdot} Y_{-} \star N\right)_{T}\right\|_{\mathbb{L}^{\frac{p}{2}}}^{\frac{p}{2}} \mathbf{1}_{p>2}+\mathbb{E}\left[\left(e^{\alpha \cdot Y_{-} \star K}\right)_{T}\right]^{+} \mathbf{1}_{p=2}\right)
$$

(iii) If $p \in(1,2)$, then for all $\varepsilon>0$ there exists $\alpha>0$ and a constant $C_{(2.10)}^{\varepsilon, \alpha}$ such that

$$
\|N\|_{\mathbb{M}^{p, \alpha}}^{p} \leq \varepsilon\left\|g^{0}\right\|_{\mathbb{H}_{1}^{p, \alpha}}^{p}+C_{(2.10)}^{\varepsilon, \alpha}\left(\|\xi\|_{\mathbb{L}^{p}}^{p}+\left\|e^{\frac{\alpha}{2} \cdot Y}\right\|_{\mathbb{S}^{p}}^{p}+\mathbb{E}\left[\left(e^{p \frac{\alpha}{2}} \cdot \phi_{p}\left(Y_{-}\right) \star K\right)_{T}\right]^{+}\right),
$$

where $\phi_{p}$ is defined in (2.7).

Proof. (i) Let us first prove (2.8). A simple application of Itô's formula implies that

$$
e^{\frac{\alpha}{2} \cdot Y .}-\int_{0}^{\cdot} e^{\frac{\alpha}{2} s}\left(g_{s}\left(Y_{s}, Z_{s}\right)+\frac{\alpha}{2} Y_{s}\right) d s
$$


is a supermartingale. Moreover, the non-decreasing process in its Doob-Meyer decomposition is $\int_{0}^{\cdot} e^{\frac{\alpha}{2} s} d K_{s}$. Therefore, Lemma A.1, Assumption 2.1 and Jensen's inequality provide

$$
\begin{aligned}
& \left(C_{\mathrm{A} .1}^{p}\right)^{-p}\|K\|_{\mathbb{I} p, \alpha}^{p} \leq\left\|e^{\frac{\alpha}{2} \cdot Y}-\int_{0}^{\cdot} e^{\frac{\alpha}{2} s}\left(g_{s}\left(Y_{s}, Z_{s}\right)+\frac{\alpha}{2} Y_{s}\right) d s\right\|_{\mathbb{S} p}^{p} \\
& \leq\left(1 \vee 2^{p-1}\right)\left(\left\|e^{\frac{\alpha}{2} \cdot Y}\right\|_{\mathbb{S} p}^{p}+\mathbb{E}\left[\left(\int_{0}^{T} e^{\frac{\alpha}{2} s}\left(\left|g_{s}\left(Y_{s}, Z_{S}\right)\right|+\frac{\alpha}{2}\left|Y_{s}\right|\right) d s\right)^{p}\right]\right) \\
& \leq\left(1 \vee 2^{p-1}\right)\left(\left(1+\left(1 \vee 3^{p-1}\right) T^{p}\left(L_{y}+\frac{\alpha}{2}\right)^{p}\right)\left\|e^{\frac{\alpha}{2}} Y\right\|_{\mathbb{S}^{p}}^{p}\right. \\
& \left.+\left(1 \vee 3^{p-1}\right)\left(L_{z}^{p}\|Z\|_{\mathbb{H} p, \alpha}^{p}+\left\|g^{0}\right\|_{\mathbb{H}_{1}^{p, \alpha}}^{p}\right)\right),
\end{aligned}
$$

in which the constant $C_{\mathrm{A} .1}^{p}$ is as in Lemma A.1.

(ii) We now turn to (2.9). As usual, we apply Itô's formula to $e^{\alpha \cdot} Y^{2}$, see [15, p. 538] for the case of làdlàg processes, use Assumption 2.1 and (2.6), to obtain

$$
\begin{aligned}
& e^{\alpha t} Y_{t}^{2}+\left(\alpha-\frac{1}{\varepsilon}-2 L_{y}-\frac{L_{z}^{2}}{\eta}\right) \int_{t}^{T} e^{\alpha s} Y_{s}^{2} d s+(1-\eta) \int_{t}^{T} e^{\alpha s}\left\|Z_{s}\right\|^{2} d s+\int_{t}^{T} e^{\alpha s} d[M-K]_{s} \\
& \leq e^{\alpha T}|\xi|^{2}+\varepsilon \int_{t}^{T} e^{\alpha s}\left|g_{s}^{0}\right|^{2} d s-2 \int_{t}^{T} e^{\alpha s} Y_{s-} d N_{s},
\end{aligned}
$$

for any $(\varepsilon, \eta) \in(0,+\infty)^{2}$. Combined with $(2.3)$, this implies that

$$
\begin{gathered}
C_{1}\|Y\|_{\mathbb{H}_{1}^{p, \alpha}}^{p}+C_{2}\|Z\|_{\mathbb{H} p, \alpha}^{p}+\|M-K\|_{\mathbb{M} p, \alpha}^{p} \\
\leq 3^{\frac{p}{2}-1}\left(e^{\frac{p}{2} \alpha T}\|\xi\|_{\mathbb{L}^{p}}^{p}+\varepsilon^{\frac{p}{2}}\left\|g^{0}\right\|_{\mathbb{H}_{1}^{p, \alpha}}^{p}\right) \\
+3^{\frac{p}{2}-1} 2^{\frac{p}{2}}\left\|\left(e^{\alpha \cdot} Y_{-} \star N\right)_{T}\right\|_{\mathbb{L}^{\frac{p}{2}}}^{\frac{p}{2}} \mathbf{1}_{p>2} \\
+3^{\frac{p}{2}-1} 2^{\frac{p}{2}} \mathbb{E}\left[\left(e^{\alpha \cdot} Y_{-} \star K\right)_{T}\right]^{+} \mathbf{1}_{p=2},
\end{gathered}
$$

where $C_{1}:=\left(\alpha-\frac{1}{\varepsilon}-2 L_{y}-\frac{L_{z}^{2}}{\eta}\right)^{\frac{p}{2}}, C_{2}:=(1-\eta)^{\frac{p}{2}}$ and where we have used Remark 2.1(iii) in the case $p=2$. Fix $\alpha>0$ and $\eta \in(0,1)$ such that $C_{1}, C_{2}>0$. We then deduce (2.9) from the right-hand side of (2.4) for $\alpha$ large enough.

(iii) It remains to prove (2.10). Since $p<2$, we can not use the Burkholder-Davis-Gundy inequality with exponent $p / 2$ to a martingale involving $M$, as it is only càdlàg. We then follow the approach proposed recently in [14]. We first appeal to Lemma A.2 below:

$$
\begin{gathered}
e^{p \frac{\alpha}{2} t}\left|Y_{t}\right|^{p}+\frac{p(p-1)}{2} \int_{t}^{T} e^{p \frac{\alpha}{2} s}\left|\phi_{p-1}\left(Y_{s}\right)\right| d[N]_{s}^{c}+\frac{\alpha p}{2} \int_{t}^{T} e^{p \frac{\alpha}{2} s}\left|Y_{s}\right|^{p} d s+A_{t}^{T} \\
\leq e^{p \frac{\alpha}{2} T}|\xi|^{p}+p \int_{t}^{T} e^{p \frac{\alpha}{2} s}\left|Y_{S}\right|^{p-1}\left|g_{s}\left(Y_{s}, Z_{s}\right)\right| d s-p \int_{t}^{T} e^{p \frac{\alpha}{2} s} \phi_{p}\left(Y_{s-}\right) d N_{s},
\end{gathered}
$$

in which

$$
A_{t}^{T}:=\frac{p(p-1)}{2} \sum_{t<s \leq T} e^{p \frac{\alpha}{2} s}\left|\Delta N_{s}\right|^{2}\left(\left|Y_{s-}\right|^{2} \vee\left|Y_{s-}+\Delta N_{s}\right|^{2}\right)^{\frac{p}{2}-1} \mathbf{1}_{\left|Y_{s-}\right| \vee\left|Y_{s-}+\Delta N_{s}\right| \neq 0},
$$


with $\Delta N:=N_{+}-N_{-}$. Recalling Assumption 2.1 and using (2.6), this shows that, for any $\beta, \gamma>0$,

$$
\begin{aligned}
& e^{p \frac{\alpha}{2} t}\left|Y_{t}\right|^{p}+\left(\frac{p(p-1)}{2}-\beta\right) \int_{t}^{T} e^{p \frac{\alpha}{2} s}\left|\phi_{p-1}\left(Y_{s}\right)\right| d[N]_{s}^{c}+p\left(\frac{\alpha}{2}-L_{y}-\frac{p L_{z}^{2}}{4 \beta}\right) \int_{t}^{T} e^{p \frac{\alpha}{2} s}\left|Y_{s}\right|^{p} d s+A_{t}^{T} \\
& \leq e^{p \frac{\alpha}{2} T}|\xi|^{p}+p \int_{t}^{T} e^{p \frac{\alpha}{2} s}\left|Y_{s}\right|^{p-1}\left|g_{s}^{0}\right| d s-p \int_{t}^{T} e^{p \frac{\alpha}{2} s} \phi_{p}\left(Y_{s-}\right) d N_{s} \\
& \leq e^{p \frac{\alpha}{2} T}|\xi|^{p}+\left\|g^{0}\right\|_{\mathbb{H}_{1}^{p, \alpha}}^{p}+\frac{p-1}{p^{\frac{p}{p-1}}} \sup _{0 \leq s \leq T}\left|e^{\frac{\alpha}{2} s} Y_{s}\right|^{p}-p \int_{t}^{T} e^{p \frac{\alpha}{2} s} \phi_{p}\left(Y_{s-}\right) d N_{s} .
\end{aligned}
$$

Let us take $\alpha \geq 2 L_{y}+p L_{z}^{2} /(2 \beta)$ with $\beta<p(p-1) / 2$. Taking expectations on both sides, we obtain

$$
\begin{aligned}
\mathbb{E}\left[\int_{t}^{T} e^{p \frac{\alpha}{2} s}\left|\phi_{p-1}\left(Y_{S}\right)\right| d[N]_{S}^{c}+A_{t}^{T}\right] \leq & C_{(2.13)}^{1}\|\xi\|_{\mathbb{L}^{p}}^{p}+C_{(2.13)}^{2}\left\|g^{0}\right\|_{\mathbb{H}_{1}^{p, \alpha}}^{p}+C_{(2.13)}^{3}\left\|e^{\frac{\alpha}{2}} \cdot Y\right\|_{\mathbb{S} p}^{p} \\
& +C_{(2.13)}^{4} \mathbb{E}\left[\left(e^{p \frac{\alpha}{2}} \phi_{p}\left(Y_{-}\right) \star K\right)_{T}\right]^{+}
\end{aligned}
$$

for some explicit constants $\left(C_{(2.13)}^{i}\right)_{1 \leq i \leq 4}$. We then argue as in [14, Step 2, Proof of Proposition 3] ${ }^{5}$ and use (2.6) again to obtain that

$$
\|N\|_{\mathbb{M}^{p, \alpha}}^{p} \leq\left(\frac{2 C_{(2.13)}^{2}}{\varepsilon p}\right)^{\frac{1}{p-1}}(2-p)\left\|e^{\frac{\alpha}{2}} Y\right\|_{\mathbb{S}^{p}}^{p}+\frac{\varepsilon}{C_{(2.13)}^{2}} \mathbb{E}\left[\int_{0}^{T} e^{p \frac{\alpha}{2} s}\left|\phi_{p-1}\left(Y_{S}\right)\right| d[N]_{S}^{c}+A_{0}^{T}\right] .
$$

We are now in position to complete the proof of Theorem 2.1.

Proof of Theorem 2.1. 1. We first assume that $p \geq 2$. In the course of this proof, we will have to choose $\alpha>0$ large to apply (2.9). However, since the norms in $\left\{\|\cdot\|_{\mathbb{H} p, \alpha}^{p}, \alpha>0\right\}$ (resp. $\left\{\|\cdot\|_{\mathbb{H}_{1}^{p, \alpha}}^{p}, \alpha>0\right\}$ and $\left\{\|\cdot\|_{\mathbb{M}^{p, \alpha}}^{p}, \alpha>0\right\}$ ) are equivalent for different values of $\alpha$, this is enough to prove our general result. We first estimate the last term in (2.9):

$$
\left\|\left(e^{\alpha \cdot} Y_{-} \star N\right)_{T}\right\|_{\mathbb{L}^{\frac{p}{2}}}^{\frac{p}{2}} \leq 2^{\frac{p}{2}-1}\left(\left\|\left(e^{\alpha \cdot} Y_{-} \star(M+Z \star W)\right)_{T}\right\|_{\mathbb{L}^{\frac{p}{2}}}^{\frac{p}{2}}+\left\|\left(e^{\alpha \cdot} Y_{-} \star K\right)_{T}\right\|_{\mathbb{L}^{\frac{p}{2}}}^{\frac{p}{2}}\right),
$$

in which, for any $\delta>0$,

$$
\left\|\left(e^{\alpha \cdot} Y_{-} \star K\right)_{T}\right\|_{\mathbb{L}^{\frac{p}{2}}}^{\frac{p}{2}} \leq \frac{1}{4 \delta}\left\|e^{\frac{\alpha}{2} \cdot} Y\right\|_{\mathbb{S} p}^{p}+\delta\|K\|_{\mathbb{I} p, \alpha}^{p},
$$

recall (2.6), and

$$
\begin{aligned}
\left\|\left(e^{\alpha \cdot} Y_{-} \star(M+Z \star W)\right)_{T}\right\|_{\mathbb{L}^{\frac{p}{2}}}^{\frac{p}{2}} & \leq C_{p}^{*} \mathbb{E}\left[\left(\int_{0}^{T} e^{2 \alpha s} Y_{s-}^{2} d[M+Z \star W]_{s}\right)^{\frac{p}{4}}\right] \\
& \leq \frac{\left(C_{p}^{*}\right)^{2}}{4 \delta}\left\|e^{\frac{\alpha}{2} \cdot} Y\right\|_{\mathbb{S}^{p}}^{p}+\delta\|M+Z \star W\|_{\mathbb{M}^{p}, \alpha}^{p},
\end{aligned}
$$

with

$$
C_{p}^{*}:=\left(\frac{p}{2} \vee \frac{p}{p-2}-1\right)^{p} \mathbf{1}_{p>2}+2 \mathbf{1}_{p=2}
$$

\footnotetext{
${ }^{5}$ As pointed out by a referee, there are some inaccuracies in the proof of Proposition 3 in [14], especially their inequality (31), which is only valid for predictable integrands. However, this inequality is never used in our proofs, and we only use similar estimates as those of their Equation (33).
} 
by Burkholder's inequality, see e.g. [22, Theorems 8.6 and 8.7]. Combining the above inequalities with (2.5) leads to

$$
\left\|\left(e^{\alpha \cdot} Y_{-} \star N\right)_{T}\right\|_{\mathbb{L}^{\frac{p}{2}}}^{\frac{p}{2}} \mathbf{1}_{p>2}+\left\|\left(e^{\alpha \cdot} Y_{-} \star K\right)_{T}\right\|_{\mathbb{L}^{\frac{p}{2}}}^{\frac{p}{2}} \mathbf{1}_{p=2} \leq \frac{C_{(2.15)}^{1}}{\delta}\left\|e^{\frac{\alpha}{2} \cdot Y}\right\|_{\mathbb{S} p}^{p}+\delta C_{(2.15)}^{2}\left(\|N\|_{\mathbb{M} p, \alpha}^{p}+\|K\|_{\mathbb{I} p, \alpha}^{p}\right),
$$

for some explicit constants $C_{(2.15)}^{1}$ and $C_{(2.15)}^{2}$ that do not depend on $\delta$. By inserting the last inequality in (2.9), for e.g. $\varepsilon=1$ and $\alpha$ chosen appropriately, we obtain for $\delta \in(0,1)$ :

$$
\left(1-\delta C_{(2.16)}^{4}\right)\|N\|_{\mathbb{M} p, \alpha}^{p} \leq C_{(2.16)}^{1}\|\xi\|_{\mathbb{L}^{p}}^{p}+C_{(2.16)}^{2}\left\|g^{0}\right\|_{\mathbb{H}_{1}^{p, \alpha}}^{p}+\frac{C_{(2.16)}^{3}}{\delta}\left\|e^{\frac{\alpha}{2} \cdot Y}\right\|_{\mathbb{S}^{p}}^{p}+\delta C_{(2.16)}^{4}\|K\|_{\mathbb{I} p, \alpha}^{p},
$$

in which the constants $\left(C_{(2.16)}^{i}\right)_{i \leq 4}$ are explicit and do not depend on $\delta \in(0,1)$. In view of the left-hand side of (2.4) and (2.8), (2.16) provides the required bound on $\|Z\|_{\mathbb{H} p, \alpha}^{p}$ by choosing $\delta>0$ small enough, so that we can then use (2.8) again to deduce the corresponding bound on $\|K\|_{\mathbb{I}, \alpha}^{p}$ :

$$
\|Z\|_{\mathbb{H}^{p, \alpha}}^{p}+\|K\|_{\mathbb{I}^{p, \alpha}}^{p} \leq C_{(2.17)}\left(\|\xi\|_{\mathbb{L}^{p}}^{p}+\|Y\|_{\mathbb{S}^{p}}^{p}+\left\|g^{0}\right\|_{\mathbb{H}_{1}^{p, \alpha}}^{p}\right),
$$

for some constant $C_{(2.17)}$. Finally, it remains to appeal to (2.5), (2.16) and (2.17) to obtain the required bound on $\|M\|_{\mathbb{M} p, \alpha}^{p}$ and conclude the proof in the case $p \geq 2$.

2. We now consider the case $p \in(1,2)$. We argue as above except that we now estimate the last term in $(2.10)$ by using (2.6):

$$
\mathbb{E}\left[\left(e^{p \frac{\alpha}{2} \cdot} \phi_{p}\left(Y_{-}\right) \star K\right)_{T}\right] \leq \frac{p-1}{\left(\delta p^{p}\right)^{\frac{1}{p-1}}}\left\|e^{\frac{\alpha}{2} \cdot Y}\right\|_{\mathbb{S} p}^{p}+\delta\|K\|_{\mathbb{I} p, \alpha}^{p} .
$$

The latter combined with (2.4), (2.5), (2.8) and (2.10), as in the end of step 1, provides the required result after choosing $\delta>0$ small enough.

When $(Y, Z, M, K)$ is only a local solution of (2.2), all the arguments above hold true after a localization. Then, using Fatou's Lemma, it follows immediately that $(Y, Z, M, K)$ is a solution. We formulate the following result but omit the proof.

Proposition 2.1. Let $(Y, Z, M, K)$ be a local solution of (2.2). Suppose in addition that $Y \in \mathbb{S}^{p}$. Then, $(Y, Z, M, K)$ is a solution of (2.2).

\subsection{Difference of solutions and stability}

In this section, we consider two terminal conditions $\xi^{1}, \xi^{2}$, as well as two generators $g^{1}$ and $g^{2}$, satisfying Assumption 2.1. We then denote by $\left(Y^{i}, Z^{i}, M^{i}, K^{i}\right) \in \mathbb{S}^{p} \times \mathbb{H}^{p} \times \mathbb{M}^{p} \times \mathbb{I}_{+}^{p}$ a solution of (2.2) with terminal condition $\xi^{i}$ and generator $g^{i}$, and set $N^{i}:=Z^{i} \star W+M^{i}-K^{i}, i=1,2$. For notational simplicity, we also define

$$
\begin{aligned}
& \delta Y:=Y^{1}-Y^{2}, \quad \delta Z:=Z^{1}-Z^{2}, \quad \delta M:=M^{1}-M^{2}, \quad \delta K:=K^{1}-K^{2}, \quad \delta N:=N^{1}-N^{2}, \\
& \delta g_{t}(\omega, y, z):=g_{t}^{1}(\omega, y, z)-g_{t}^{2}(\omega, y, z), \quad \text { for all }(t, \omega, y, z) \in[0, T] \times \Omega \times \mathbb{R} \times \mathbb{R}^{d} .
\end{aligned}
$$

By Assumption 2.1, we know that there is an $\mathbb{R}$-valued (resp. $\mathbb{R}^{d}$-valued), $\mathbb{F}$-progressively measurable process $\lambda$ (resp. $\eta$ ), with $|\lambda| \leq L_{y}$ (resp. $\|\eta\| \leq L_{z}$ ) such that

$$
\delta g_{t}:=g_{t}^{1}\left(Y_{t}^{1}, Z_{t}^{1}\right)-g_{t}^{2}\left(Y_{t}^{2}, Z_{t}^{2}\right)=\delta g_{t}\left(Y_{t}^{1}, Z_{t}^{1}\right)+\lambda_{t} \delta Y_{t}+\eta_{t} \cdot \delta Z_{t} .
$$

Then, $(\delta Y, \delta Z, \delta M, \delta K)$ satisfies (2.2) with driver $\delta g$ and terminal condition $\delta \xi$. In particular, we can apply to it the results of Remark 2.1 and Lemma 2.1 .

The main result of this section, Theorem 2.2 below, is in the spirit of Theorem 2.1: it suffices to control the norm of $\delta Y$ to control the norms of $\delta Z$ and $\delta(M-K)$. Seemingly, it should just be an application of Theorem 2.1 to $(\delta Y, \delta Z, \delta M, \delta K)$ as it satisfies an equation of the form (2.2). However, it is not the case: 
(i) In Theorem 2.2, we will only control $\delta(M-K)$ and not $\delta M$ and $\delta K$ separately. Actually, as shown in Example A. 1 below, there is no hope to control these two processes separately even in the seemingly benign case where $g^{1}=g^{2}=0$.

(ii) Actually, Theorem 2.2 can not be an immediate consequence of Theorem 2.1, because the process $\delta K$ which appears in the dynamics of $\delta Y$ is no longer non-decreasing, and more importantly because the result of Lemma A.1 below does not hold for quasimartingales (instead of supermartingales). However, it is a direct consequence of the intermediate estimates of Lemma 2.1, which explains why they have been isolated.

(iii) If one has a more precise knowledge of the behavior of the non-decreasing processes $K^{1}$ and $K^{2}$, then these estimates can actually be improved. We will make this point more clear when we will treat the special case of reflected BSDEs in Section 3.

Let us now state our result.

Theorem 2.2. For any $\alpha \geq 0$, there is a constant $C_{2.2}^{\alpha}$ such that

$$
\|\delta Z\|_{\mathbb{H} p, \alpha}^{p}+\|\delta(M-K)\|_{\mathbb{M} p, \alpha}^{p} \leq C_{2.2}^{\alpha}\left(\|\delta \xi\|_{\mathbb{L}^{p}}^{p}+\|\delta Y\|_{\mathbb{S} p}^{p}+\|\delta Y\|_{\mathbb{S} p}^{\frac{p}{2} \wedge(p-1)}+\left\|\delta g\left(Y_{.}^{1}, Z_{.}^{1}\right)\right\|_{\mathbb{H}_{1}^{p, \alpha}}^{p}\right) .
$$

The constant $C_{2.2}^{\alpha}$ depends on $L_{y}, L_{z}, p$ and $\alpha$, as well as $\left(\left\|Y^{i}\right\|_{\mathbb{S} p}^{p},\left\|\xi^{i}\right\|_{\mathbb{L}^{p}}^{p},\left\|g^{i}(0,0)\right\|_{\mathbb{H}_{1}^{p, \alpha}}^{p}\right)_{i=1,2}$.

Proof. In this proof, we take $\alpha$ large enough so as to apply the estimates of Lemma 2.1. The general case is deduced by recalling that the different norms are equivalent for different values of $\alpha$, since $[0, T]$ is compact.

1 . We first assume that $p \geq 2$. We apply (2.9) to $(\delta Y, \delta Z, \delta M, \delta K)$ and obtain

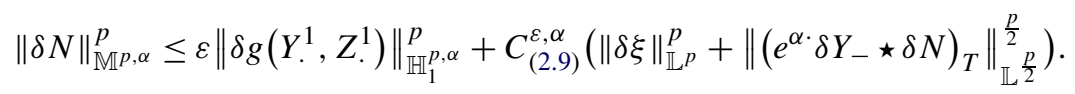

Let us estimate the last term in this inequality. We remind the reader that $\delta N=\delta Z \star W+\delta M-\delta K$. We first use (2.3) to obtain

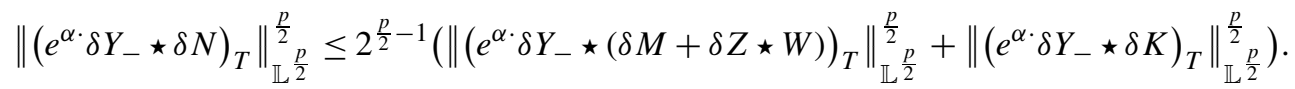

We then apply Burkholder inequality and obtain

$$
\begin{aligned}
\left\|\left(e^{\alpha \cdot} \delta Y_{-} \star(\delta M+\delta Z \star W)\right)_{T}\right\|_{\mathbb{L}^{\frac{p}{2}}}^{\frac{p}{2}} & \leq C_{p}^{*}\left\|e^{\frac{\alpha}{2}} \cdot \delta Y\right\|_{\mathbb{S} p}^{\frac{p}{2}}\|\delta M+\delta Z \star W\|_{\mathbb{H} p, \alpha}^{\frac{p}{2}} \\
& \leq 2^{\frac{p-2}{4}} C_{p}^{*}\left\|e^{\frac{\alpha}{2}} \delta Y\right\|_{\mathbb{S}^{p}}^{\frac{p}{2}} \sum_{i=1}^{2}\left\|M^{i}+Z^{i} \star W\right\|_{\mathbb{H} p, \alpha}^{\frac{p}{2}},
\end{aligned}
$$

where $C_{p}^{*}$ is as in (2.14), while

$$
\left\|\left(e^{\alpha \cdot \delta} Y_{-} \star \delta K\right)_{T}\right\|_{\mathbb{L}^{\frac{p}{2}}}^{\frac{p}{2}} \leq\left\|e^{\frac{\alpha}{2}} \cdot \delta Y\right\|_{\mathbb{S}^{p}}^{\frac{p}{2}}\|\delta K\|_{\mathbb{P}^{p, \alpha}}^{\frac{p}{2}} \leq 2^{\frac{p-1}{2}}\left\|e^{\frac{\alpha}{2}} \cdot \delta Y\right\|_{\mathbb{S}^{p}}^{\frac{p}{2}} \sum_{i=1}^{2}\left\|K^{i}\right\|_{\mathbb{I}^{p, \alpha}}^{\frac{p}{2}} .
$$

We can then conclude the proof in the case $p \geq 2$ by using (2.4) and the bounds of Theorem 2.1 applied to $\left(Z^{i}, M^{i}, K^{i}\right)_{i=1,2}$.

2. We now assume that $p \in(1,2)$ and proceed as above but use (2.10) in place of (2.9). Namely, since

$$
\mathbb{E}\left[\left(e^{p \frac{\alpha}{2} \cdot} \phi_{p}\left(\delta Y_{-}\right) \star \delta K\right)_{T}\right] \leq\left\|e^{\frac{\alpha}{2} \cdot \delta Y}\right\|_{\mathbb{S}^{p}}^{p-1}\|\delta K\|_{\mathbb{I} p, \alpha} \leq\left\|e^{\frac{\alpha}{2} \cdot \delta Y}\right\|_{\mathbb{S}^{p}}^{p-1} \sum_{i=1}^{2}\left\|K^{i}\right\|_{\mathbb{I} p, \alpha},
$$

it suffices to use (2.4) and the bound of Theorem 2.1 applied to $K^{1}$ and $K^{2}$. 


\section{Application to reflected BSDEs: A general existence result}

The results of the previous section show that it suffices to control the norm of $Y$ (resp. $\delta Y$ ) in order to control the norm of $(Z, M, K)$ (resp. $(\delta Z, \delta M, \delta K))$, given a solution $(Y, Z, M, K)$ (resp. two solutions $\left(Y^{1}, Z^{1}, M^{1}, K^{1}\right)$ and $\left(Y^{2}, Z^{2}, M^{2}, K^{2}\right)$ ) of (2.2). In most examples of applications, we know how to control the norm of $Y$ (and $\delta Y$ ). This is in particular the case in the context of reflected BSDEs (see e.g. [8]), BSDEs with constraints (see e.g. [5,25]), 2nd order BSDE (see e.g. [27,29]), weak BSDEs (see [1]).

Let us exemplify this in the context of reflected BSDEs. In particular, the following results extend Klimsiak [1113] to a filtration that only satisfies the usual conditions, and may not be quasi left-continuous. For sake of simplicity, we restrict to the case of a càdlàg obstacle, see [10] and the references therein for the additional specific arguments that could be used for irregular obstacles. Recall that $\mathbb{S}_{r}^{p}$ (resp. $\mathbb{I}_{r}^{p}, \mathbb{I}_{+, r}^{p}$ ) denote the set of elements of $\mathbb{S}^{p}$ (resp. $\mathbb{I}^{p}$, $\mathbb{I}_{+}^{p}$ ) with càdlàg path, $\mathbb{P}$-a.s.

\subsection{A priori estimates for reflected BSDEs}

In this section, we assume that Assumption 2.1 holds for $\xi$ and $g$.

Definition 3.1. Let $S$ be a càdlàg process such that $S^{+}:=S \vee 0 \in \mathbb{S}_{r}^{p}$. We say that $(Y, Z, M, K) \in \mathbb{S}_{r}^{p} \times \mathbb{H}^{p} \times \mathbb{M}^{p} \times$ $\mathbb{I}_{+, r}^{p}$ is a solution of the reflected BSDE with lower obstacle $S$ if

$$
Y_{t}=\xi-\int_{t}^{T} g_{s}\left(Y_{s}, Z_{s}\right) d s-\int_{t}^{T} Z_{s} \cdot d W_{s}-\int_{t}^{T} d M_{s}+K_{T}-K_{t},
$$

holds for any $t \in[0, T] \mathbb{P}$-a.s., and if

$$
\begin{cases}Y_{t} \geq S_{t}, & t \in[0, T], \mathbb{P} \text {-a.s., } \\ \int_{0}^{T}\left(Y_{s-}-S_{s-}\right) d K_{s}=0, & \mathbb{P} \text {-a.s. (Skorokhod condition). }\end{cases}
$$

In order to provide a first estimate on the component $Y$ of a solution, we use the classical linearization procedure. By Assumption 2.1, there exists a $\mathbb{R}$-valued (resp. $\mathbb{R}^{d}$-valued), $\mathbb{F}$-progressively measurable (resp. $\mathbb{F}$-predictable) process $\lambda$ (resp. $\eta$ ), with $|\lambda| \leq L_{y}$ (resp. $\|\eta\| \leq L_{z}$ ) such that

$$
g_{s}\left(Y_{s}, Z_{s}\right)=g_{s}^{0}+\lambda_{s} Y_{s}+\eta_{s} \cdot Z_{s}, \quad s \in[0, T]
$$

Let us define

$$
X_{s}:=e^{-\int_{0}^{s} \lambda_{s} d s}, \quad \text { and } \quad \frac{d \mathbb{Q}}{d \mathbb{P}}:=\mathcal{E}\left(-\int_{0}^{\cdot} \eta_{s} \cdot d W_{s}\right)_{T}, \quad W^{\mathbb{Q}}:=W .+\int_{0}^{\cdot} \eta_{s} d s,
$$

in which $\mathcal{E}$ denotes the Doléans-Dade exponential. Then, by Girsanov theorem, $W^{\mathbb{Q}}$ is a $\mathbb{Q}$-Brownian motion, $M$ is still a $\mathbb{Q}$-martingale orthogonal to $W^{\mathbb{Q}}$, and we can re-write the solution of the reflected BSDE (3.1) as

$$
\begin{cases}X_{t} Y_{t}=X_{T} \xi-\int_{t}^{T} X_{s} g_{s}^{0} d s-\int_{t}^{T} X_{s} Z_{s} \cdot d W_{s}^{\mathbb{Q}}-\int_{t}^{T} X_{s-} d M_{s}+\int_{t}^{T} X_{s-} d K_{s}, & t \in[0, T], \\ X_{t} Y_{t} \geq X_{t} S_{t}, & t \in[0, T], \\ \int_{0}^{T} X_{s-}\left(Y_{s-}-S_{s-}\right) d K_{s}=0 . & \end{cases}
$$

One can now use the link between reflected BSDEs and optimal stopping problems. The proof is classical so that we omit it, see [8] for a proof in a Brownian filtration and for a continuous obstacle, or [17] for a càdlàg obstacle, and [6] for more results on optimal stopping. We denote by $\mathcal{T}_{t, T}$ the set of $[t, T]$-valued $\mathbb{F}$-stopping times, while $\mathbb{E}_{t}$ and $\mathbb{E}_{t}^{\mathbb{Q}}$ stands for the $\mathcal{F}_{t}$-conditional expectations under $\mathbb{P}$ and $\mathbb{Q}$. 
Proposition 3.1. Let $(Y, Z, M, K)$ and $S$ be as in Definition 3.1. Then,

$$
X_{t} Y_{t}=\underset{\tau \in \mathcal{T}_{t, T}}{\operatorname{ess} \sup } \mathbb{E}_{t}^{\mathbb{Q}}\left[-\int_{t}^{\tau} X_{s} g_{s}^{0} d s+X_{\tau} S_{\tau} \mathbf{1}_{\tau<T}+X_{T} \xi \mathbf{1}_{\tau=T}\right],
$$

and

$$
Y_{t}=\underset{\tau \in \mathcal{T}_{t, T}}{\operatorname{ess} \sup } \mathbb{E}_{t}\left[-\int_{t}^{\tau} g_{s}\left(Y_{s}, Z_{s}\right) d s+S_{\tau} \mathbf{1}_{\tau<T}+\xi \mathbf{1}_{\tau=T}\right],
$$

for all $t \leq T$.

Before continuing, let us introduce the solution $(\mathcal{Y}, \mathcal{Z}, \mathcal{M}) \in \mathbb{S}_{r}^{p} \times \mathbb{H}^{p} \times \mathbb{M}^{p}$ of the following BSDE (wellposedness is a direct consequence of Theorem 4.1 below, see also [14]),

$$
\mathcal{Y}_{t}=\xi-\int_{t}^{T} g_{s}\left(\mathcal{Y}_{s}, \mathcal{Z}_{s}\right) d s-\int_{t}^{T} \mathcal{Z}_{s} \cdot d W_{s}-\int_{t}^{T} d \mathcal{M}_{s}, \quad t \in[0, T], \mathbb{P} \text {-a.s. }
$$

A simple application of the comparison result, which can be proved as in [14, Proposition 4], implies that

$$
Y_{t} \geq \mathcal{Y}_{t}, \quad t \in[0, T], \mathbb{P} \text {-a.s. }
$$

Let us first show that Proposition 3.1 is actually enough to control the $Y$-term of a solution (or the $\delta Y$-term of the difference of two solutions) and therefore that Theorems 2.1 and 2.2 apply to reflected BSDEs.

Proposition 3.2. (i) Let $(Y, Z, M, K)$ and $S$ be as in Definition 3.1. Then, for any $\alpha \geq 0$,

$$
\left\|e^{\frac{\alpha}{2} \cdot Y}\right\|_{\mathbb{S} p}^{p} \leq C_{3.2}^{\alpha}\left(\|\xi\|_{\mathbb{L}^{p}}^{p}+\left\|e^{L_{y} \cdot} S^{+}\right\|_{\mathbb{S} p}^{p}+\mathbb{E}\left[\left(\int_{0}^{T} e^{L_{y} s}\left|g_{s}^{0}\right| d s\right)^{p}\right]\right)+C_{\mathcal{Y}}^{\alpha}\left\|e^{\frac{\alpha}{2} \cdot \mathcal{Y}}\right\|_{\mathbb{S} p}^{p},
$$

for some constants $C_{3.2}^{\alpha}$ and $C_{\mathcal{Y}}^{\alpha}$ that only depend on $L_{y}, L_{z}$ and $\alpha$. Moreover, if we replace $S^{+}$by $S$ in the above, we can take $C_{\mathcal{Y}}^{\alpha}=0$.

(ii) For $i=1,2$, let $\left(Y^{i}, Z^{i}, M^{i}, K^{i}\right)$ and $S^{i}$ be as in Definition 3.1 for a generator $g^{i}$ satisfying Assumption 2.1 and terminal condition $\xi^{i} \in \mathbb{L}^{p}$. Then, for any $\alpha \geq 0$,

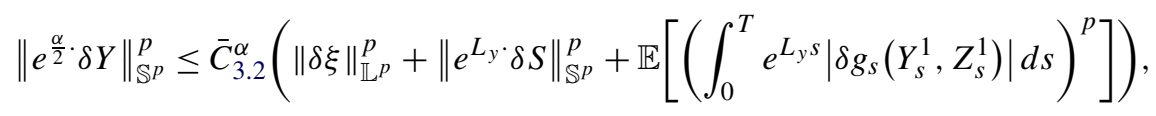

for some constant $\bar{C}_{3.2}^{\alpha}$ that only depends on $L_{y}, L_{z}$ and $\alpha .^{6}$

Proof. (i) First of all, we recall that $Y \geq \mathcal{Y}$ on $[0, T]$. Next, we deduce from Proposition 3.1 that, for any $1<\kappa<p$,

$$
\begin{aligned}
& \sup _{t \in[0, T]}\left\{e^{\frac{\alpha}{2} t}\left|Y_{t}\right|\right\} \\
& \leq \sup _{t \in[0, T]} e^{\left(L_{y}+\frac{\alpha}{2}\right) t} \mathbb{E}_{t}^{\mathbb{Q}}\left[\int_{0}^{T} e^{L_{y} s}\left|g_{s}^{0}\right| d s+\sup _{s \in[0, T]}\left\{e^{L_{y} s} S_{s}^{+}\right\}+e^{L_{y} T}|\xi|\right]+\sup _{t \in[0, T]}\left\{e^{\frac{\alpha}{2} t}\left|\mathcal{Y}_{t}\right|\right\} \\
& =\sup _{t \in[0, T]} e^{\left(L_{y}+\frac{\alpha}{2}\right) t} \mathbb{E}_{t}\left[\mathcal{E}\left(-\int_{t}^{T} \eta_{s} \cdot d W_{s}\right)\left(\int_{0}^{T} e^{L_{y} s}\left|g_{s}^{0}\right| d s+\sup _{s \in[0, T]}\left\{e^{L_{y} s} S_{s}^{+}\right\}+e^{L_{y} T}|\xi|\right)\right] \\
& \quad+\sup _{t \in[0, T]}\left\{e^{\frac{\alpha}{2} t}\left|\mathcal{Y}_{t}\right|\right\}
\end{aligned}
$$

\footnotetext{
${ }^{6}$ We are grateful to Marie-Claire Quenez for indicating us a technical problem in the proof in the first version.
} 


$$
\begin{aligned}
\leq & e^{\left(L_{y}+\frac{\alpha}{2}\right) T+\frac{\kappa}{2(\kappa-1)} L_{z}^{2} T} 3^{\frac{\kappa-1}{\kappa}} \sup _{t \in[0, T]}\left(\mathbb{E}_{t}\left[\left(\int_{0}^{T} e^{L_{y} s}\left|g_{s}^{0}\right| d s\right)^{\kappa}+\sup _{s \in[0, T]}\left\{e^{\kappa L_{y} s}\left(S_{s}^{+}\right)^{\kappa}\right\}+e^{\kappa L_{y} T}|\xi|^{\kappa}\right]\right)^{\frac{1}{\kappa}} \\
& +\sup _{t \in[0, T]}\left\{e^{\frac{\alpha}{2} t}\left|\mathcal{Y}_{t}\right|\right\},
\end{aligned}
$$

in which it is clear that we could have suppressed the term involving $\mathcal{Y}$ if we had used $S$ instead of $S^{+}$.

Hence, we deduce from Doob's inequality with the exponent $p / \kappa$ and (2.3) that

$$
\begin{aligned}
\left\|e^{\frac{\alpha}{2}} Y\right\|_{\mathbb{S} p}^{p} \leq & e^{p\left(L_{y}+\frac{\alpha}{2}\right) T+p \frac{\kappa}{2(\kappa-1)} L_{z}^{2} T} 6^{p-1}\left(\frac{p}{p-\kappa}\right)^{\frac{p}{\kappa}} \mathbb{E}\left[\left(\int_{0}^{T} e^{L_{y} s}\left|g_{s}^{0}\right| d s\right)^{p}\right] \\
& +e^{p\left(L_{y}+\frac{\alpha}{2}\right) T+p \frac{\kappa}{2(\kappa-1)} L_{z}^{2} T} 6^{p-1}\left(\frac{p}{p-\kappa}\right)^{\frac{p}{\kappa}}\left(\left\|e^{L_{y} \cdot} S^{+}\right\|_{\mathbb{S}^{p}}^{p}+e^{p L_{y} T}\|\xi\|_{\mathbb{L}^{p}}^{p}\right)+2^{p-1}\left\|e^{\frac{\alpha}{2}} \mathcal{Y}\right\|_{\mathbb{S} p}^{p},
\end{aligned}
$$

where we have to replace the $6^{p-1}$ by $3^{p-1}$ if we use $S$ instead of $S^{+}$.

(ii) We first use a classical argument. We know that there exists an $\mathbb{R}$-valued (resp. $\mathbb{R}^{d}$-valued), $\mathbb{F}$-progressively measurable process $\widetilde{\lambda}($ resp. $\widetilde{\eta})$, with $|\widetilde{\lambda}| \leq L_{y}$ (resp. $\|\widetilde{\eta}\| \leq L_{z}$ ) such that

$$
g_{s}^{1}\left(Y_{s}^{1}, Z_{s}^{1}\right)-g_{s}^{2}\left(Y_{s}^{2}, Z_{s}^{2}\right)=\delta g_{s}\left(Y_{s}^{1}, Z_{s}^{1}\right)+\tilde{\lambda}_{s} \delta Y_{s}+\tilde{\eta}_{s} \cdot \delta Z_{s}, \quad s \in[0, T], \mathbb{P} \text {-a.s. }
$$

Therefore, we can define $\tilde{\mathbb{Q}} \sim \mathbb{P}$ and a bounded positive process $\tilde{X}$ as in (3.2) above such that

$$
\tilde{X}_{t} \delta Y_{t}=\mathbb{E}_{t}^{\tilde{\mathbb{Q}}}\left[\int_{t}^{\tau} \tilde{X}_{s} \delta g_{s}\left(Y_{s}^{1}, Z_{s}^{1}\right) d s+\tilde{X}_{\tau}\left(Y_{\tau}^{1}-Y_{\tau}^{2}\right)+\int_{t}^{\tau} \tilde{X}_{s} d\left(K^{1}-K^{2}\right)_{s}\right],
$$

for all stopping time $\tau \geq t$. Set $\tau_{\varepsilon}:=\inf \left\{s \geq t: Y_{s}^{1} \leq S_{s}^{1}+\varepsilon\right\} \wedge T$. Clearly, $Y_{-}^{1} \geq S_{-}^{1}+\varepsilon$ on [[t, $\left.\left.\tau_{\varepsilon}\right]\right]$. Hence, $K_{\tau_{\varepsilon}}^{1}-$ $K_{t}^{1}=0$ by the Skorokhod condition. Moreover, $Y_{\tau_{\varepsilon}}^{1} \leq S_{\tau_{\varepsilon}}^{1}+\varepsilon$ on $\left\{\tau_{\varepsilon}<T\right\}$. Then, the above leads to

$$
\begin{aligned}
\tilde{X}_{t} \delta Y_{t} & \leq \mathbb{E}_{t}^{\tilde{\mathbb{Q}}}\left[\int_{t}^{\tau_{\varepsilon}} \tilde{X}_{s}\left|\delta g_{s}\left(Y_{s}^{1}, Z_{s}^{1}\right)\right| d s+\tilde{X}_{\tau_{\varepsilon}}\left(Y_{\tau_{\varepsilon}}^{1}-Y_{\tau_{\varepsilon}}^{2}\right)+0-\int_{t}^{\tau_{\varepsilon}} \tilde{X}_{s} d K_{s}^{2}\right] \\
& \leq \underset{\tau \in \mathcal{T}_{t, T}}{\operatorname{ess} \sup } \mathbb{E}_{t}^{\tilde{\mathbb{Q}}}\left[\int_{t}^{\tau} \tilde{X}_{s}\left|\delta g_{s}\left(Y_{s}^{1}, Z_{s}^{1}\right)\right| d s+\tilde{X}_{\tau}\left|\delta S_{\tau}\right| \mathbf{1}_{\tau<T}+\tilde{X}_{T}|\delta \xi| \mathbf{1}_{\tau=T}+\varepsilon \tilde{X}_{\tau}\right] .
\end{aligned}
$$

Since the same applies to $Y^{2}-Y^{1}$ in place of $\delta Y=Y^{1}-Y^{2}$, it follows that

$$
\tilde{X}_{t}\left|\delta Y_{t}\right| \leq \underset{\tau \in \mathcal{T}_{t, T}}{\operatorname{ess} \sup } \mathbb{E}_{t}^{\tilde{\mathbb{Q}}}\left[\int_{t}^{\tau} \tilde{X}_{s}\left|\delta g_{s}\left(Y_{s}^{1}, Z_{s}^{1}\right)\right| d s+\tilde{X}_{\tau}\left|\delta S_{\tau}\right| \mathbf{1}_{\tau<T}+\tilde{X}_{T}|\delta \xi| \mathbf{1}_{\tau=T}+\varepsilon \tilde{X}_{\tau}\right] .
$$

It remains to let $\varepsilon$ go to 0 and then argue exactly as in (i).

We now show that one can actually take advantage of the Skorokhod condition satisfied by the solution of a reflected BSDE to improve the general stability result of Theorem 2.2. This result is crucial in order to prove existence of a solution when $p$ is arbitrary, see the proof of Theorem 3.1 below. We only provide the result for $p=2$. It could be extended to $p \in(1,2)$, but this is not important as we can always reduce to $p=2$ by localization, again see the proof of Theorem 3.1 below.

Proposition 3.3. For $i=1,2$, let $\left(Y^{i}, Z^{i}, M^{i}, K^{i}\right)$ be as in (ii) of Proposition 3.2. Then, for any $\varepsilon>0$, there exists $\alpha$ large enough such that

$$
\|\delta Y\|_{\mathbb{H}_{1}^{2, \alpha}}^{2}+\|\delta Z\|_{\mathbb{H}^{2, \alpha}}^{2}+\|\delta(M-K)\|_{\mathbb{M}^{2, \alpha}}^{2} \leq \varepsilon\left\|\delta g\left(Y_{.}^{1}, Z_{.}^{1}\right)\right\|_{\mathbb{H}_{1}^{2, \alpha}}^{2}+C_{3.3}^{\alpha}\left(\|\delta \xi\|_{L^{2}}^{2}+\left\|e^{\frac{\alpha}{2}} \cdot \delta S\right\|_{\mathbb{S}^{2}}\right),
$$

for some constant $C_{3.3}^{\alpha}$ that only depends on $L_{y}, L_{g}, \alpha, \varepsilon$ and $\left(\left\|Y^{i}\right\|_{\mathbb{S}^{2}}^{2},\left\|\xi^{i}\right\|_{\mathbb{L}^{2}}^{2},\left\|g^{i}(0,0)\right\|_{\mathbb{H}_{1}^{2, \alpha}}^{2}\right)_{i=1,2}$. 
Proof. It suffices to apply (2.4), (2.9) to $(\delta Y, \delta Z, \delta M, \delta K)$ and use the Skorokhod condition to deduce the control

$$
\mathbb{E}\left[\int_{t}^{T} e^{\alpha s} \delta Y_{s-} d\left(\delta K_{s}\right)\right] \leq \mathbb{E}\left[\int_{t}^{T} e^{\alpha s} \delta S_{s-} d\left(\delta K_{S}\right)\right] \leq\left\|e^{\frac{\alpha}{2}} \delta S\right\|_{\mathbb{S}^{2}}\|\delta K\|_{\mathbb{I}^{2, \alpha}},
$$

in which $\|\delta K\|_{\mathbb{I}^{2, \alpha}}$ is bounded by Theorem 2.1 and Proposition 3.2.

\subsection{Wellposedness of reflected BSDE under general filtration}

We can finally prove the existence of a unique solution to the reflected BSDEs (3.1). Our proof is extremely close to the original one given in [8], but relies on the more general estimates given in this paper.

Theorem 3.1. Let Assumption 2.1 hold true. Then, there is a unique solution $(Y, Z, M, K)$ to the reflected BSDE (3.1).

Proof. The uniqueness is an immediate consequence of Proposition 3.3, recall that the Doob-Meyer decomposition of the supermartingale $M-K$ is unique. We therefore concentrate on the problem of existence. In the following, we let $(\mathcal{Y}, \mathcal{Z}, \mathcal{M}) \in \mathbb{S}_{r}^{p} \times \mathbb{H}^{p} \times \mathbb{M}^{p}$ denote the unique solution to the BSDE (3.3) with generator $g$ and terminal condition $\xi$.

1. First, let us consider the case $p=2$ and prove the existence in 3 steps.

(i) We assume that the function $g$ does not depend on $(y, z)$, that is, $g_{t}(\omega, y, z)=g_{t}(\omega)$. Using [13, Theorem 2.12], there are adapted càdlàg processes $(Y, M, K)$ and $Z \in \mathbb{H}_{\text {loc }}^{1}$, such that $M$ is a martingale, $K$ is non-decreasing, and (3.1) holds true. Moreover, [13, Corollary 2.8] implies

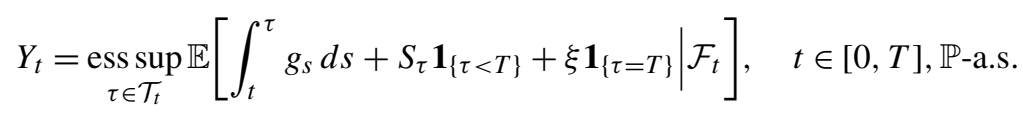

Hence,

$$
\left|Y_{t}\right| \leq \mathbb{E}\left[|\xi|+\int_{0}^{T}\left|g_{s}\right| d s+\sup _{0 \leq s \leq T} S_{s}^{+}+\sup _{0 \leq s \leq T}\left|\mathcal{Y}_{s}\right| \mid \mathcal{F}_{t}\right]
$$

by the above equation combined with (3.4), and the Burkholder-Davis-Gundy inequality can be used in conjunction with Theorem 4.1 below to deduce that

$$
\|Y\|_{\mathbb{S}^{2}}^{2} \leq C\left(\|\xi\|_{\mathbb{L}^{2}}^{2}+\|g\|_{\mathbb{H}_{1}^{2}}+\left\|S^{+}\right\|_{\mathbb{S}^{2}}^{2}\right)<\infty,
$$

for some $C>0$. By Theorem 2.1, it follows that $(Z, M, K) \in \mathbb{H}^{2} \times \mathbb{M}^{2} \times \mathbb{I}_{+, r}^{2}$, and hence $(Y, Z, M, K)$ is the unique solution to (3.1) by Proposition 3.2.

(ii) We now consider the general case. Given $\alpha>0$, let us consider the space $\mathcal{S}$ of processes $(Y, Z)$ such that $(Y, Z) \in \mathbb{S}_{r}^{2} \times \mathbb{H}^{2, \alpha}$ and denote

$$
\|(Y, Z)\|_{2, \alpha}:=\left\|e^{\alpha \cdot} Y\right\|_{\mathbb{S}^{2}}+\|Z\|_{\mathbb{H}^{2, \alpha}} .
$$

Clearly, $\left(\mathcal{S},\|\cdot\|_{2, \alpha}\right)$ is a Banach space. Then, existence can be proved by using the classical fixed point argument. Let us set $\left(\bar{Y}^{0}, \bar{Z}^{0}, \bar{M}^{0}, \bar{K}^{0}\right):=(0,0,0,0)$ and define $\left(\bar{Y}^{n}, \bar{Z}^{n}, \bar{M}^{n}, \bar{K}^{n}\right)_{n \geq 1}$ recursively as the solution to the following reflected BSDE:

$$
\bar{Y}_{t}^{n+1}=\xi-\int_{t}^{T} g_{s}\left(\bar{Y}_{s}^{n}, \bar{Z}_{s}^{n}\right) d s-\int_{t}^{T} \bar{Z}_{s}^{n+1} \cdot d W_{s}-\int_{t}^{T} d \bar{M}_{s}^{n+1}+\int_{t}^{T} d \bar{K}^{n+1},
$$

with

$$
\bar{Y}_{t}^{n+1} \geq S_{t}, \quad t \in[0, T] \quad \text { and } \quad \int_{0}^{T}\left(\bar{Y}_{t-}^{n+1}-S_{t-}\right) d K_{t}^{n+1}=0, \quad \mathbb{P} \text {-a.s. }
$$


Well-posedness is ensured by the first step above. Indeed, the only thing we have to check is that $g\left(\bar{Y}_{.}^{n}, \bar{Z}^{n}\right) \in \mathbb{H}_{1}^{2}$. However, this is a direct consequence of the fact that $g^{0} \in \mathbb{H}_{1}^{2}$, that $g$ is uniformly Lipschitz continuous in $(y, z)$, and that $\left(\bar{Y}^{n}, \bar{Z}^{n}\right) \in \mathbb{S}^{2} \times \mathbb{H}^{2}$ by induction.

Let us also denote $\bar{L}^{n}:=\bar{M}^{n}-\bar{K}^{n}$. Using the estimates of Proposition 3.3, it follows that, for $\alpha>0$ large enough, $\left(\bar{Y}^{n}, \bar{Z}^{n}\right)_{n \geq 1}$ is a Cauchy sequence in $\mathbb{H}_{1}^{2, \alpha} \times \mathbb{H}^{2, \alpha}$, and hence a Cauchy sequence in $\mathbb{S}_{r}^{2} \times \mathbb{H}^{2, \alpha}$ by the estimates in Part (ii) of Proposition 3.2. Moreover, by (3.5), we have

$$
\begin{aligned}
\bar{L}_{t}^{n}-\bar{L}_{t}^{m}= & \left(\bar{Y}_{t}^{n}-\bar{Y}_{t}^{m}\right)-\left(\bar{Y}_{0}^{n}-\bar{Y}_{0}^{m}\right)-\int_{0}^{t}\left(\bar{Z}_{s}^{n}-\bar{Z}_{s}^{m}\right) \cdot d W_{s} \\
& -\int_{0}^{t}\left(g_{s}\left(\bar{Y}_{s}^{n-1}, \bar{Z}_{s}^{n-1}\right)-g_{s}\left(\bar{Y}_{s}^{m-1}, \bar{Z}_{s}^{m-1}\right)\right) d s .
\end{aligned}
$$

It follows that $\left(\bar{Y}^{n}, \bar{Z}^{n}, \bar{L}^{n}\right)_{n \geq 1}$ is a Cauchy sequence in $\left(\mathcal{S} \times \mathbb{S}^{2},\|\cdot\|_{2, \alpha}+\|\cdot\|_{\mathbb{S}^{2}}\right)$, from which we can pass to the limit and obtain

$$
\bar{Y}_{t}=\xi-\int_{t}^{T} g_{s}\left(\bar{Y}_{s}, \bar{Z}_{s}\right) d s-\int_{t}^{T} \bar{Z}_{s} \cdot d W_{s}-\int_{t}^{T} d \bar{L}_{s}
$$

as well as $\bar{Y} \geq S$.

(iii) We now prove that $L$ is a supermartingale having the decomposition $\bar{L}=: \bar{M}-\bar{K}$ where $\bar{M}$ is orthogonal to $W$ and where the non-decreasing process $K$ satisfies the Skorokhod condition, that is to say that we can pass to the limit in (3.6). First, the fact that $L$ is a càdlàg supermartingale is immediate from the convergence of $\bar{L}^{n}$ in $\mathbb{S}^{2}$ and the dominated convergence theorem. The fact that the brackets $\left[\bar{L}^{n}, W\right]$ converge to $[\bar{L}, W]$ is clear from, for instance Corollaire 1.9 in [19] or the proof of Proposition 2 in [4], which proves the orthogonality of $\bar{L}$ and $W$.

Let now $\bar{L}=\bar{M}-\bar{K}$ be its Doob-Meyer decomposition, and let us consider a sequence of stopping times $\left(\tau_{m}\right)_{m \geq 1}$ such that the process $\sup _{n \geq 1}\left(\bar{Y}^{n}-S\right) \mathbf{1}_{\left[\left[0, \tau_{m}[[\right.\right.}$ is essentially bounded and $\tau_{m} \longrightarrow \infty$ as $m \longrightarrow \infty$. Since $\left\|\bar{Y}^{n}-\bar{Y}\right\|_{\mathbb{S}^{2}}+$ $\left\|\bar{L}^{n}-\bar{L}\right\|_{\mathbb{S}^{2}} \longrightarrow 0$, it follows that

$$
-\mathbb{E}\left[\int_{0}^{\tau_{m}}\left(\bar{Y}_{t-}-S_{t-}\right) d \bar{K}_{t}\right]=\mathbb{E}\left[\int_{0}^{\tau_{m}}\left(\bar{Y}_{t-}-S_{t-}\right) d \bar{L}_{t}\right]=\lim _{n \rightarrow \infty} \mathbb{E}\left[\int_{0}^{\tau_{m}}\left(\bar{Y}_{t-}^{n}-S_{t-}\right) d \bar{L}_{t}^{n}\right]=0 .
$$

Since $\bar{K}$ is non-decreasing and $\bar{Y} \geq S$ on $[0, T]$, we thus obtain

$$
\int_{0}^{\tau_{m}}\left(\bar{Y}_{t-}-S_{t-}\right) d \bar{K}_{t}=0, \quad \mathbb{P} \text {-a.s. }
$$

Letting $m \longrightarrow \infty$, we see that the Skorokhod condition (3.6) holds true for $\bar{K}$.

2. Finally, let us consider the general case when $p \in(1, \infty)$. It follows from (3.4) that one can replace $S$ by $S \vee \mathcal{Y}$, and therefore reduce to the case where $S \in \mathbb{S}_{r}^{p}$ (and not only $S^{+} \in \mathbb{S}_{r}^{p}$ ), which we assume in the following. In this case, we can define for $n \geq 1$

$$
\xi^{n}:=(-n) \vee \xi \wedge n, \quad S^{n}:=(-n) \vee S \wedge n, \quad \text { and } \quad g^{n}:=(-n) \vee g \wedge n,
$$

so that $\left(\xi^{n}, S^{n}, g^{n}(0,0)\right)_{n \geq 1} \in \mathbb{L}^{2} \times \mathbb{S}_{r}^{2} \times \mathbb{H}_{1}^{2, \alpha}$, for any $\alpha \geq 0$. Thus by Step 1 , we know that there is a unique solution $\left(Y^{n}, Z^{n}, M^{n}, K^{n}\right) \in \mathbb{S}_{r}^{2} \times \mathbb{H}^{2} \times \mathbb{M}^{2} \times \mathbb{I}_{+, r}^{2}$ to (3.1). Since $\left(\xi^{n}, S^{n}, g^{n}(0,0)\right)_{n \geq 1}$ is a Cauchy sequence in $\mathbb{L}^{p} \times \mathbb{S}_{r}^{p} \times$ $\mathbb{H}_{1}^{p, \alpha}$, the estimates of Proposition 3.2 and Theorem 2.2 show that the sequence $\left(Y^{n}, Z^{n}\right)_{n \geq 1}$ is also a Cauchy sequence in $\mathbb{S}_{r}^{p} \times \mathbb{H}^{p, \alpha}$, for any $\alpha \geq 0$. Moreover, by a similar equality as in (3.7), it follows that $\left(L^{n}:=M^{n}-K^{n}\right)_{n \geq 1}$ is also a Cauchy sequence in $\mathbb{S}_{r}^{p}$. Using the same arguments as in (iii) of Step 1, it is easy to check that its limit is a solution to $(3.1)$. 


\section{Side remark}

Note that the existence and uniqueness of a solution $(Y, Z, M) \in \mathbb{S}_{r}^{p} \times \mathbb{H}^{p} \times \mathbb{M}^{p}$ to the $\mathrm{BSDE}$

$$
Y_{t}=\xi-\int_{t}^{T} g_{s}\left(Y_{s}, Z_{s}\right) d s-\int_{t}^{T} Z_{s} \cdot d W_{s}-\int_{t}^{T} d M_{s}
$$

follows from the same arguments as the one used in Section 3 whenever $\xi \in \mathbb{L}^{p}$ and $g^{0} \in \mathbb{H}_{1}^{p}$. Indeed, we can bound the component $Y$ of a solution as in the proof of Proposition 3.2 by

$$
X_{t} Y_{t}=\mathbb{E}_{t}^{\mathbb{Q}}\left[-\int_{t}^{T} X_{s} g_{s}^{0} d s+X_{T} \xi\right], \quad \mathbb{P} \text {-a.s. }
$$

in which $X$ and $\mathbb{Q}$ are defined as in (3.2). The difference of the $Y$-components of two solutions can be bounded similarly. Then, it suffices to apply the same fixed point argument as in the proof of Theorem 3.1.

Theorem 4.1. Let Assumption 2.1 hold true. Then, (4.1) admits a unique solution in $\mathbb{S}_{r}^{p} \times \mathbb{H}^{p} \times \mathbb{M}^{p}$. Moreover, for all $\alpha \geq 0$, there exists a constant $C_{4.1}^{\alpha}$ that depends only on $L_{y}, L_{z}$ and $\alpha$, such that

$$
\left\|e^{\frac{\alpha}{2} \cdot Y}\right\|_{\mathbb{S} p}^{p} \leq C_{4.1}^{\alpha}\left(\|\xi\|_{\mathbb{L}^{p}}^{p}+\mathbb{E}\left[\left(\int_{0}^{T} e^{L_{y} s}\left|g_{s}^{0}\right| d s\right)^{p}\right]\right)
$$

Remark 4.1. Recalling that the difference of two solutions, with different terminal conditions and generators, is still a solution to a BSDE, the bound of Theorem 4.1 applies. Using similar notations as above, we have:

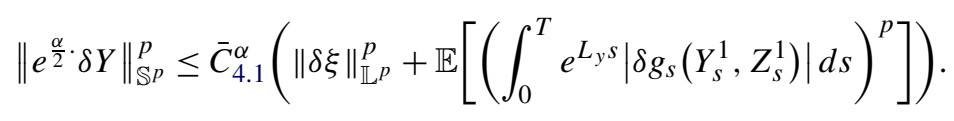

\section{Appendix}

Let us consider a strong supermartingale $X \in \mathbb{S}^{p}$ on $[0, T]$. Then, its paths are almost surely làdlàg, and it admits the (unique) Doob-Meyer decomposition (see e.g. Mertens [20]):

$$
X_{t}=X_{0}+M_{t}-A_{t}-I_{t},
$$

where $M$ is a right-continuous martingale, with $M_{0}=0, A$ is a predictable non-decreasing right-continuous process with $A_{0}=0$, and $I$ is a predictable non-decreasing left-continuous process with $I_{0}=0$.

The following extends [16, Theorem 3.1], which is the key ingredient of our main results, Theorem 2.1 and Theorem 2.2.

Lemma A.1. For every constant $p>1$, there is some constant $C_{A .1}^{p}>0$ such that, for all strong supermartingale $X \in \mathbb{S}^{p}$ (with the decomposition (A.1)), one has

$$
\|A\|_{\mathbb{I} p}+\|I\|_{\mathbb{I} p} \leq C_{\mathrm{A} .1}^{p}\|X\|_{\mathbb{S} p} .
$$

Proof. (i) We suppose in addition that $X$ is right-continuous so that $I \equiv 0$. Denote $X^{*}:=\sup _{0 \leq t \leq T}\left|X_{t}\right|$ and define $\tilde{X}$ as the right-continuous version of the martingale $\mathbb{E}\left[X^{*} \mid \mathcal{F}_{t}\right]$. Then $\widehat{X}:=X+\tilde{X}$ is a non-negative right-continuous supermartingale on $[0, T]$, with the Doob-Meyer decomposition

$$
\widehat{X}_{t}=X_{0}+\left(\tilde{X}_{t}+M_{t}\right)-A_{t} .
$$


Setting $\widehat{X}_{t}:=\widehat{X}_{T}$ for $t \in[T, T+1)$ and $\widehat{X}_{t}=0$ for $t \in[T+1, \infty)$, then $\widehat{X}$ is in fact a right-continuous potential (recall that a potential is a non-negative right-continuous supermartingale $\widehat{X}$ on $[0, \infty)$ such that $\lim _{t \rightarrow \infty} \mathbb{E}\left[\widehat{X}_{t}\right]=0$ ). Using Meyer [21, Theorem 1] (see also [16, Theorem 3.1]), there is some constant $C_{p}^{\prime}$ such that

$$
\|A\|_{\mathbb{I} p} \leq C_{p}^{\prime}\|\widehat{X}\|_{\mathbb{S} p} .
$$

By the definition of $\widehat{X}$ and Doob's martingale inequality, we get

$$
\|A\|_{\mathbb{I} p} \leq C_{p}^{\prime}\|\widehat{X}\|_{\mathbb{S} p} \leq C_{p}^{\prime}\left(1+\frac{p}{p-1}\right)\|X\|_{\mathbb{S} p} .
$$

(ii) We now consider the case when $X$ is not necessary right-continuous. By Mertens [20], we know that the process $X+I$ is a right-continuous strong supermartingale, and that the left-continuous process $I$ is obtained as the limit of an increasing sequence $I .:=\lim _{\varepsilon \rightarrow 0} \lim _{n \rightarrow \infty} I^{\varepsilon, n}$, where $I^{\varepsilon, n}$ is defined by

$$
I_{t}^{\varepsilon, n}:=\sum_{k=1}^{n}\left(X_{\sigma_{k}^{\varepsilon, n}}-X_{\sigma_{k}^{\varepsilon, n}+}\right) \mathbf{1}_{\sigma_{k}^{\varepsilon, n}<t},
$$

in which $\left(\sigma_{k}^{\varepsilon, n}\right)_{1 \leq k \leq n}$ is the non-decreasing sequence of stopping times which exhausts the first $n$ jumps of $X$ such that $X_{t}-X_{t+} \geq \varepsilon$, i.e.

$$
\sigma_{1}^{\varepsilon, n}:=\inf \left\{t \geq 0: X_{t}-X_{t+} \geq \varepsilon\right\}, \quad \sigma_{k+1}^{\varepsilon, n}:=\inf \left\{t>\sigma_{k}^{\varepsilon, n}: X_{t}-X_{t+} \geq \varepsilon\right\}, \quad k=1, \ldots, n-1 .
$$

In Step (iii) we will show that $\left\|I^{\varepsilon, n}\right\|_{\mathbb{I}^{p}} \leq C_{p}^{\prime \prime}\|X\|_{\mathbb{S}^{p}}$ for some constant $C_{p}^{\prime \prime}>0$ independent of $\varepsilon$ and $n$, then it follows from the monotone convergence theorem that $\|I\|_{\mathbb{I} p} \leq C_{p}^{\prime \prime}\|X\|_{\mathbb{S} p}$, and hence

$$
\|A\|_{\mathbb{I} p} \leq C_{p}^{\prime}\left(1+\frac{p}{p-1}\right)\|X+I\|_{\mathbb{S} p} \leq C_{p}^{\prime}\left(1+\frac{p}{p-1}\right)\left(1+C_{p}^{\prime \prime}\right)\|X\|_{\mathbb{S} p} .
$$

(iii) It is now enough to prove that $\left\|I^{\varepsilon, n}\right\|_{\mathbb{I}^{p}} \leq C_{p}^{\prime \prime}\|X\|_{\mathbb{S} p}$ for some constant $C_{p}^{\prime \prime}$ independent of $(n, \varepsilon)$. Notice that the discrete process $\bar{X}^{n}:=\left(X_{0}, X_{\sigma_{1}^{\varepsilon, n}}, X_{\sigma_{1}^{\varepsilon, n}+}, \ldots, X_{\sigma_{n}^{\varepsilon, n}}, X_{\sigma_{n}^{\varepsilon, n}+}\right)$ is a discrete time supermartingale. By interpolation, we can turn it into a right-continuous strong supermartingale on $[0, T]$. Then, using the results in Step (i) we obtain that

$$
\left\|I^{\varepsilon, n}\right\|_{\mathbb{I}^{p}} \leq C_{p}^{\prime \prime}\|X\|_{\mathbb{S} p}, \quad \text { with } C_{p}^{\prime \prime}:=C_{p}^{\prime}\left(1+\frac{p}{p-1}\right) .
$$

Remark A.1. A careful reading of the proof in [21] shows that the constant can be computed explicitly and is given by

$$
C_{p}^{\prime}:=\min _{2 \leq k<p}\left(p \prod_{j=2}^{k} \frac{p j}{p-j}\right)^{\frac{k}{p-1}} \mathbf{1}_{p>2}+\left(\frac{p^{2}}{p-1}\right)^{\frac{1}{p-1}} \mathbf{1}_{p \in(1,2]}
$$

Meyer's result show that for a supermartingale $X$ with Doob-Meyer's decomposition $X=X_{0}+M-A$, we can control $A$ by $X$; the following example shows that given two supermartingales, we cannot control the difference of the $A$ parts by the difference of the supermartingales.

Example A.1. Let $W$ be a one dimensional Brownian motion. Fix $\varepsilon>0$ and let $V$ be defined by

$$
V_{t}:=\sum_{k \geq 0} W_{\tau_{k}} \mathbf{1}_{\left[\tau_{k}, \tau_{k+1}\right)}(t), \quad \text { where } \tau_{0}:=0, \tau_{k+1}:=\inf \left\{t \geq \tau_{k}:\left|W_{t}-W_{\tau_{k}}\right| \geq \varepsilon\right\} .
$$


Notice that $V$ is of finite variation with decomposition $V=V^{+}-V^{-}$such that $V^{+}$and $V^{-}$are two non-negative nondecreasing and predictable process. Let $X^{1}:=W-V^{+}$and $X^{2}:=-V^{-}$, then $\sup _{t}\left|X_{t}^{1}-X_{t}^{2}\right|=\sup _{t}\left|W_{t}-V_{t}\right| \leq \varepsilon$, but $V^{+}-V^{-}=V$ cannot be controlled by $\varepsilon$.

We finally provide a technical lemma used in the paper. Recall the definition of $\phi_{p}$ in (2.7) and observe that $\left|\phi_{p-1}(y)\right|=|y|^{p-2} \mathbf{1}_{y \neq 0}$.

Lemma A.2. Let $X$ be a làdlàg semimartingale. Then for all $p \in(1,2)$ and $\alpha>0$, we have $\mathbb{P}$-a.s. for any $t \in[0, T]$

$$
\begin{aligned}
e^{p \frac{\alpha}{2} t}\left|X_{t}\right|^{p} \leq & e^{p \frac{\alpha}{2} T}\left|X_{T}\right|^{p}-p \int_{t}^{T} e^{p \frac{\alpha}{2} s} \frac{\alpha}{2}\left|X_{s}\right|^{p} d s-p \int_{t}^{T} e^{p \frac{\alpha}{2} s} \phi_{p}\left(X_{s-}\right) d X_{s} \\
& -\frac{p(p-1)}{2} \int_{t}^{T} e^{p \frac{\alpha}{2} s}\left|\phi_{p-1}\left(X_{s}\right)\right| d[X]_{s}^{c} \\
& -\frac{p(p-1)}{2} \sum_{t<s \leq T} e^{p \frac{\alpha}{2} s}\left|X_{s+}-X_{s-}\right|^{2}\left(\left|X_{s^{-}}\right|^{2} \vee\left|X_{s+}\right|^{2}\right)^{\frac{p}{2}-1} \mathbf{1}_{\left|X_{s-}\right| \vee\left|X_{s+}\right| \neq 0},
\end{aligned}
$$

where we denote $X_{T+}:=X_{T}$.

Proof. This is an immediate consequence of a straightforward adaptation of [14, Lemmas 7, 8 and 9], together with the Itô's formula for làdlàg processes in [15, p. 538].

\section{References}

[1] B. Bouchard, R. Élie and A. Réveillac. BSDEs with weak terminal condition. Ann. Probab. 43 (2) (2015) 572-604. MR3306000

[2] B. Bouchard, D. Possamaï and X. Tan. A general Doob-Meyer-Mertens decomposition for $g$-supermartingale systems. Electron. J. Probab. 21 (36) (2015) 1-21. MR3508683

[3] P. Briand, B. Delyon, Y. Hu, É. Pardoux and L. Stoica. $L^{p}$ solutions of backward stochastic differential equations. Stochastic Process. Appl. 108 (2003) 109-129. MR2008603

[4] P. Briand, B. Delyon and J. Mémin. On the robustness of backward stochastic differential equations. Stochastic Process. Appl. 97 (2002) 229-253. MR1875334

[5] J. Cvitanić, I. Karatzas and H. M. Soner. Backward stochastic differential equations with constraints on the gain-process. Ann. Probab. 26 (1998) 1522-1551. MR1675035

[6] N. El Karoui. Les aspects probabilistes du contrôle stochastique. In Ecole d'Eté de Probabilités de Saint-Flour IX-1979 73-238. Lecture Notes in Mathematics 876, 1981. MR0637471

[7] N. El Karoui and S.-J. Huang. A general result of existence and uniqueness of backward stochastic differential equations. In Backward Stochastic Differential Equations (Paris, 1995-1996) 27-36. Pitman Res. Notes Math. Ser. 364. Longman, Harlow, 1997. MR1752673

[8] N. El Karoui, C. Kapoudjian, E. Pardoux, S. Peng and M.-C. Quenez. Reflected solutions of backward SDE's, and related obstacle problems for PDE's. Ann. Appl. Probab. 25 (2) (1997) 702-737. MR1434123

[9] N. El Karoui, S. Peng and M.-C. Quenez. Backward stochastic differential equations in finance. Math. Finance 7 (1) (1997) 1-71. MR1434407

[10] M. Grigorova, P. Imkeller, E. Offen, Y. Ouknine and M.-C. Quenez. Reflected BSDEs when the obstacle is not right-continuous and optimal stopping. Preprint, 2015. Available at arXiv:1504.06094.

[11] T. Klimsiak. BSDEs with monotone generator and two irregular reflecting barriers. Bull. Sci. Math. 137 (3) (2013) 268-321. MR3043077

[12] T. Klimsiak. Reflected BSDEs with monotone generator. Electron. J. Probab. 17 (107) (2014) 1-25. MR3015691

[13] T. Klimsiak. Reflected BSDEs on filtered probability spaces. Stochastic Process. Appl. 125 (11) (2015) 4204-4241. MR3385601

[14] T. Kruse and A. Popier. BSDEs with monotone generator driven by Brownian and Poisson noises in a general filtration. Stoch. Int. J. Probab. Stoch. Process. 88 (4) (2016) 491-539. MR3473849

[15] É. Lenglart. Tribus de Meyer et théorie des processus. Séminaire de probabilité (Strasbourg) 14 (1980) 500-546. MR0580151

[16] É. Lenglart, D. Lépingle and M. Pratelli. Présentation unifiée de certaines inégalités de la théorie des martingales. Séminaire de probabilités (Strasbourg) 14 (1980) 26-48. MR0580107

[17] J. P. Lepeltier and M. Xu. Penalization method for reflected backward stochastic differential equations with one r.c.1.1. barrier. Statist. Probab. Lett. 75 (1) (2005) 58-66. MR2185610

[18] Q. Lin and S. Peng. Smallest g-supersolutions for BSDE with continuous drift coefficients. Chin. Ann. Math. Ser. B 21B (3) (2000) 359-366. MR1788292

[19] J. Mémin and L. Słomiński. Condition UT et stabilité en loi des solutions d'équations différentielles stochastiques. Séminaire de probabilités (Strasbourg) 25 (1991) 162-177. MR1187779 
[20] J.-F. Mertens. Théorie des processus stochastiques généraux applications, aux surmartingales. Probab. Theory Related Fields 22 (1) (1972) 45-68. MR0346895

[21] P.-A. Meyer. Une majoration du processus croissant naturel associé à une surmartingale. Séminaire de probabilités (Strasbourg) 2 (1968) 166-170. MR0242240

[22] A. Osȩkowski. Sharp Martingale and Semimartingale Inequalities. Birkhaüser, Basel, 2012. MR2964297

[23] S. Peng. Monotonic limit theorem of BSDE and nonlinear decomposition theorem of Doob-Meyer's type. Probab. Theory Related Fields 113 (4) (1999) 473-499. MR1717527

[24] S. Peng and $\mathrm{M}$. Xu. The smallest $g$-supermartingale and reflected BSDE with single and double $L^{2}$ obstacles. Ann. Inst. Henri Poincaré Probab. Stat. 41 (2005) 605-630. MR2139035

[25] S. Peng and M. Xu. Constrained BSDE and viscosity solutions of variation inequalities. Preprint, 2007. Available at arXiv:0712.0306.

[26] S. Peng and M. Xu. Reflected BSDE with a constraint and its applications in an incomplete market. Bernoulli 16 (3) (2010) 614-640. MR2730642

[27] D. Possamaï, X. Tan and C. Zhou. Stochastic control for a class of nonlinear stochastic kernels and applications. Preprint, 2015. Available at arXiv: 1510.08439.

[28] M. Schweizer. Approximating random variables by stochastic integrals. Ann. Appl. Probab. 22 (3) (1994) 1536-1575. MR1303653

[29] M. Soner, N. Touzi and J. Zhang. Wellposedness of second order backward SDEs. Probab. Theory Related Fields 153 (1-2) (2012) 149-190. MR2925572 